Reduced-cost second-order algebraic-diagrammatic construction method for excitation energies and transition moments

Dávid Mester, Péter R. Nagy, and Mihály Kállay

Citation: The Journal of Chemical Physics 148, 094111 (2018); doi: 10.1063/1.5021832

View online: https://doi.org/10.1063/1.5021832

View Table of Contents: http://aip.scitation.org/toc/jcp/148/9

Published by the American Institute of Physics

AIP| The Jouminal Pal Physics

PERSPECTIVES 


\title{
Reduced-cost second-order algebraic-diagrammatic construction method for excitation energies and transition moments
}

\author{
Dávid Mester,a) Péter R. Nagy, and Mihály Kállay ${ }^{b)}$ \\ MTA-BME Lendület Quantum Chemistry Research Group, Department of Physical Chemistry \\ and Materials Science, Budapest University of Technology and Economics, P.O. Box 91, \\ H-1521 Budapest, Hungary
}

(Received 9 January 2018; accepted 19 February 2018; published online 7 March 2018)

\begin{abstract}
A reduced-cost implementation of the second-order algebraic-diagrammatic construction [ADC(2)] method is presented. We introduce approximations by restricting virtual natural orbitals and natural auxiliary functions, which results, on average, in more than an order of magnitude speedup compared to conventional, density-fitting $\operatorname{ADC}(2)$ algorithms. The present scheme is the successor of our previous approach [D. Mester, P. R. Nagy, and M. Kállay, J. Chem. Phys. 146, 194102 (2017)], which has been successfully applied to obtain singlet excitation energies with the linear-response second-order coupled-cluster singles and doubles model. Here we report further methodological improvements and the extension of the method to compute singlet and triplet ADC(2) excitation energies and transition moments. The various approximations are carefully benchmarked, and conservative truncation thresholds are selected which guarantee errors much smaller than the intrinsic error of the $\mathrm{ADC}(2)$ method. Using the canonical values as reference, we find that the mean absolute error for both singlet and triplet $\mathrm{ADC}(2)$ excitation energies is $0.02 \mathrm{eV}$, while that for oscillator strengths is 0.001 a.u. The rigorous cutoff parameters together with the significantly reduced operation count and storage requirements allow us to obtain accurate $\operatorname{ADC}(2)$ excitation energies and transition properties using triple- $\zeta$ basis sets for systems of up to one hundred atoms. Published by AIP Publishing. https://doi.org/10.1063/1.5021832
\end{abstract}

\section{INTRODUCTION}

There are several, nowadays, actively researched phenomena related to the excited electronic states of molecular systems. For instance, excited states play an important role for photochromic materials, for photo-initialized chemical processes, and for energy transfer and storage. Quantum-chemical methods have now become routine tools in the investigation of excited-state properties and processes. Consequently, it is important to develop efficient but reliable methods for the excited states of extended molecular systems.

Many theories have been developed in the past few decades to investigate excited-state and transition properties. These are, for example, the time-dependent density functional theory (TD-DFT) ${ }^{1,2}$ as well as the wave function-based semi-empirical ${ }^{3-6}$ and ab initio methods. ${ }^{7-27}$ Presently, for the investigation of extended systems with more than 1500 basis functions, a TD-DFT approach is the most common choice. However, the limits of TD-DFT have been identified previously for challenging cases, ${ }^{28,29}$ such as Rydberg and charge transfer (CT) states, or $\pi \rightarrow \pi^{*}$ excitations of a conjugated system. The correlated wave function methods usually provide a more reliable option for small cases. Excited states can simply be treated by the multi-configurational

a)Electronic mail: mester.david@mail.bme.hu

b)Electronic mail: kallay@mail.bme.hu self-consistent field (MCSCF $)^{30}$ method, by the multireference configuration interaction, ${ }^{31}$ or with the various propagator-based schemes. ${ }^{13,14}$ Excited-state theories have also been developed for coupled-cluster (CC) approaches invoking the equation-of-motion $(\mathrm{EOM})^{7,8}$ and the linearresponse $(\mathrm{LR})^{9-11}$ techniques. However, the much higher computational demand of such methods is often a limiting factor in practice. Some of the most affordable methods, such as the complete active space SCF (CASSCF) ${ }^{15}$ approach, the second-order $\mathrm{CC}$ singles and doubles (CC2) method, ${ }^{16-21}$ the second-order polarization propagator approximation (SOPPA) ${ }^{22-24}$ method, and the second-order algebraic-diagrammatic construction $[\mathrm{ADC}(2)]^{25}$ approach have already been applied to realistic, but relatively small systems.

Among the theories suitable for excited-state property calculations, ADC is one of the most promising approaches. It is a Hermitian and size-consistent method, and it is relatively easy to implement. The ADC scheme was first derived by Schirmer ${ }^{25}$ employing a diagrammatic perturbation expansion of the polarization propagator, utilizing the Møller-Plesset partitioning of the Hamiltonian. A similar result was later obtained with the so-called intermediate state representation (ISR) approach developed by Schirmer et al. ${ }^{32-34}$ While the initial implementations of the theory were limited to its secondorder variant $[\mathrm{ADC}(2)]$, later it was extended to the third-order $[\mathrm{ADC}(3)]{ }^{26,27} \mathrm{~A}$ more efficient implementation ${ }^{35-38}$ of the $\mathrm{ADC}(2)$ and $\mathrm{ADC}(3)$ methods and extensive benchmark calculations ${ }^{36}$ were reported by Dreuw et al. In these studies, 
the performance of $\mathrm{ADC}(2)$ was also compared to that of the closely related but more demanding $\mathrm{CC} 2$ approach, and it has been proven that the $\mathrm{ADC}(2)$ method is practically as accurate as CC2. ${ }^{36,39}$ Furthermore, tools to compute twophoton absorption, ${ }^{40}$ static polarizability, ${ }^{41}$ core-valence excitations, ${ }^{42}$ and excited-state dynamics ${ }^{43}$ were also developed. The ADC method was also combined with the spin-flip, ${ }^{44}$ the scaled-opposite-spin, ${ }^{39}$ and the frozen density embedding ${ }^{45}$ approaches.

The ADC(2) method is often used to study the excitedstate properties of molecular systems; however, as it scales as the fifth power of the system size, the upper limit of its applicability is around 30 heavy atoms or 1500 basis functions. For more extended systems, instead of using the less accurate TD-DFT methods, an alternative solution may be the reduction of the computational costs of the ADC(2) method. One of the most commonly used approximations for that purpose is the density fitting (DF) approach which was introduced by Shavitt et al. ${ }^{46}$ and further developed by Whitten ${ }^{47}$ and Dunlap and co-workers. ${ }^{48}$ In the DF approach, the four-center electron repulsion integrals are written in an approximate form as the products of two- and three-center integrals; therefore, the operation count, the memory requirement, and the number of the input-output (I/O) operations can be greatly reduced. ${ }^{19,49}$ As it was demonstrated by Hättig, if the DF is combined with spinscaling and Laplace transform techniques, the fifth-order scaling of $\mathrm{ADC}(2)$ can be reduced to fourth-order. ${ }^{50}$ A promising new approach for the rank reduction of higher-order quantities, such as the two-electron integral or the doubles coefficient tensors, is the tensor hypercontraction (THC) scheme of Martínez, Sherrill, and their co-workers. ${ }^{51-53}$ It was demonstrated that the THC approach can reduce the scaling of $\mathrm{CC} 2$ and potentially that of $\mathrm{ADC}(2)$ to fourth-order even if the full exchange term is included. ${ }^{54,55}$

Another widely used technique to reduce the computational costs is to restrict the subspace in which the equations are solved. One of the simplest approaches is the restricted virtual space approach, where the canonical virtual orbitals with orbital energies higher than a predetermined threshold are neglected. The method was also employed for excited-state CC models, ${ }^{56,57}$ while its applicability for ADC approaches was recently demonstrated by Sundholm et al..$^{58,59}$ as well as Yang and Dreuw. ${ }^{60} \mathrm{~A}$ further possibility for reducing the subspace is the introduction of local approximations used often nowadays, the basic idea of which comes from Pulay and coworkers. ${ }^{61,62}$ Concerning the ADC(2) method, Schütz ${ }^{63}$ and Helmich and Hättig ${ }^{64}$ developed localized molecular orbital (MO)-based approaches, while local schemes for calculating excitation energies and oscillator strengths with the related linear response (LR) $\mathrm{CC} 2$ method have been proposed by Schütz and co-workers ${ }^{65-70}$ as well as by Baudin and Kristensen. ${ }^{71,72}$ In the case of the frozen natural orbital (NO) approximation, a one-particle density matrix is constructed and diagonalized. Of the resulting NOs, those ones are retained which have large occupation numbers, i.e., eigenvalues, and are supposed to give a significant contribution to the electron correlation. ${ }^{73-75}$ While the approximation is widely used for ground-state correlation methods, ${ }^{76-79}$ its use for excitedstate calculations are rather limited. ${ }^{80,81}$ A closely related approach, the quasiparticle virtual orbital scheme, was developed by Ortiz and co-workers for the cost reduction of electron propagator methods. ${ }^{82-84}$ The natural auxiliary function (NAF) approach, which was introduced by one of us ${ }^{85}$ is similar to the NO approximation. In this case, the size of the fitting basis is reduced in a similar way as for the abovementioned method. To the best of our knowledge, the ADC variants of the NO and NAF approximations have not been developed.

In this paper, our NO- and NAF-based approach ${ }^{86}$ is extended to the $\mathrm{ADC}(2)$ method. We report an improved version of the previous algorithm which is more robust and enables significantly faster calculations. We also extend the considered excited-state properties to triplet excitation energies and transition moments. We assess the accuracy of the approach in detail and carry out calculations for organic dyes of various sizes.

\section{THEORY}

\section{A. The ADC(2) method}

The ground-state $\mathrm{ADC}(2)$ correlation energy is simply obtained from second-order Møller-Plesset (MP2) perturbation theory as

$$
\Delta E_{\mathrm{MP} 2}=\sum_{i j a b}(i a \mid j b)\left(2 t_{i j}^{a b}-t_{j i}^{a b}\right),
$$

where $i, j, \ldots(a, b, \ldots)$ denote occupied (virtual) spatial molecular orbital (MO) indices. Later, $p, q, \ldots$ will be used as general MO indices. The above first-order amplitudes, $t_{i j}^{a b}$, are given in the canonical Hartree-Fock (HF) basis as

$$
t_{i j}^{a b}=\frac{(i a \mid j b)}{\varepsilon_{i}+\varepsilon_{j}-\varepsilon_{a}-\varepsilon_{b}}=\frac{(i a \mid j b)}{D_{i j}^{a b}},
$$

where $\varepsilon_{i}\left(\varepsilon_{a}\right)$ is the occupied (virtual) orbital energy and (ialjb) denotes a two-electron integral using the Mulliken notation. Utilizing this, the first-order Møller-Plesset (MP1) wave function reads as

$$
\left|\Psi^{\mathrm{MP} 1}\right\rangle=\left(1+T_{2}\right)|0\rangle,
$$

where $|0\rangle$ is the HF determinant. Here the double excitations are described by $T_{2}$ in the following form:

$$
T_{2}=\frac{1}{2} \sum_{a i b j} t_{i j}^{a b} E_{a i} E_{b j}=\frac{1}{2} \sum_{\mu_{2}} t_{\mu_{2}} \tau_{\mu_{2}},
$$

where we have introduced a shorthand notation for the excitation operator, $\tau_{\mu_{2}}=E_{a i} E_{b j}$, which is constructed from spincoupled one-particle excitation operators $E_{a i}=a_{\alpha}^{+} i_{\alpha}^{-}+a_{\beta}^{+} i_{\beta}^{-}$ with creation operators $a_{\eta}^{+}, b_{\eta}^{+}, \ldots$ and annihilation operators $i_{\eta}^{-}, j_{\eta}^{-}, \ldots$ for spin orbitals with $\eta$ spin.

The ADC(2) ansatz for the wave function of the excited states is given in the form of

$$
\left|\Psi^{\mathrm{ADC}(2)}\right\rangle=\left(R_{1}+R_{2}\right)\left|\Psi^{\mathrm{MP} 1}\right\rangle,
$$


where the spin-coupled single and double excitation operators, $R_{1}$ and $R_{2}$, respectively, can be defined similar to Eq. (4) with $r_{\mu_{1}}$ and $r_{\mu_{2}}$ as the corresponding coefficients.
The excitation energy, being correct up to second-order, is obtained via the diagonalization of the following Hermitian Jacobian:

$$
\mathbf{A}^{\mathrm{ADC}(2)}=\left(\begin{array}{cc}
\frac{1}{2}\left(\left\langle\mu_{1}\left|\left[\left[H, T_{2}\right], \tau_{v_{1}}\right]\right| 0\right\rangle+\left\langle v_{1}\left|\left[\left[H, T_{2}\right], \tau_{\mu_{1}}\right]\right| 0\right\rangle\right) & \left\langle\mu_{1}\left|\left[H, \tau_{v_{2}}\right]\right| 0\right\rangle \\
\left\langle\mu_{2}\left|\left[H, \tau_{v_{1}}\right]\right| 0\right\rangle & \left\langle\mu_{2}\left|\left[F, \tau_{v_{2}}\right]\right| 0\right\rangle
\end{array}\right),
$$

where $\left|\mu_{n}\right\rangle$ stands for $n$-fold excited configurations and $F$ is the Fock-operator. Similar to the case of linear-response secondorder coupled-cluster singles and doubles (LR-CC2), ${ }^{19}$ in practice, the

$$
\sigma=\mathbf{A}^{\mathrm{ADC}(2)} \mathbf{r}=\omega_{\mathrm{ADC}(2)} \mathbf{r}
$$

eigenvalue problem is recast as a non-linear eigenvalue equation

$$
\sigma\left(\omega_{\mathrm{ADC}(2)}, \mathbf{r}_{1}\right)=\mathbf{A}^{\mathrm{eff}}\left(\omega_{\mathrm{ADC}(2)}\right) \mathbf{r}_{1}=\omega_{\mathrm{ADC}(2)} \mathbf{r}_{1},
$$

where $\omega_{\mathrm{ADC}(2)}$ is the $\mathrm{ADC}(2)$ excitation energy and $\mathbf{r}_{1}(\mathbf{r})$ is a vector composed of the $r_{\mu_{1}}\left(r_{\mu_{1}}\right.$ and $\left.r_{\mu_{2}}\right)$ coefficients. The benefit is that the resulting equation with the effective Jacobian matrix $\mathbf{A}^{\text {eff }}\left(\omega_{\mathrm{ADC}(2)}\right)$ has to be solved only for the $r_{\mu_{1}}$ amplitudes corresponding to single excitations. The elements of the effective Jacobian read explicitly as

$$
A_{\mu_{1} \nu_{1}}^{\mathrm{eff}}\left(\omega_{\mathrm{ADC}(2)}\right)=A_{\mu_{1} v_{1}}-\sum_{\gamma_{2}} \frac{A_{\mu_{1} \gamma_{2}} A_{\gamma_{2} \nu_{1}}}{\varepsilon_{\gamma_{2}}-\omega_{\mathrm{ADC}(2)}}
$$

with $\varepsilon_{\gamma_{2}}=-D_{i j}^{a b}$ if $\tau_{\gamma_{2}}=E_{a i} E_{b j}$.

In the following, we briefly collect the working equations required for the implementation of the $\operatorname{ADC}(2)$ method in spatial MO basis because, to the best of our knowledge, they are not published in the literature. Deriving the expressions corresponding to Eq. (8) we arrive at the

$$
\begin{aligned}
\sigma_{i a}= & \sum_{j b}[2(i a \mid j b)-(i j \mid a b)] r_{j}^{b}+\left(\varepsilon_{a}-\varepsilon_{i}\right) r_{i}^{a}+\frac{1}{2} \sum_{k j b c}[2(k c \mid j b)-(j c \mid k b)] r_{k}^{c}\left(2 t_{i j}^{a b}-t_{j i}^{a b}\right)+\frac{1}{2} \sum_{k j b c}[2(i a \mid j b)-(j a \mid i b)] r_{k}^{c}\left(2 t_{k j}^{c b}-t_{j k}^{c b}\right) \\
& +\frac{1}{2} \sum_{k j b c}(i b \mid c k)\left(2 t_{j k}^{b c}-t_{k j}^{b c}\right) r_{j}^{a}+\frac{1}{2} \sum_{k j b c}(j b \mid c k)\left(2 t_{i k}^{b c}-t_{k i}^{b c}\right) r_{j}^{a}-\frac{1}{2} \sum_{k j b c}(j b \mid c k)\left(2 t_{j k}^{a c}-t_{k j}^{a c}\right) r_{i}^{b}-\frac{1}{2} \sum_{k j b c}(j a \mid c k)\left(2 t_{j k}^{b c}-t_{k j}^{b c}\right) r_{i}^{b} \\
& +\sum_{b k c}(a b \mid c k) \hat{R}_{i k}^{b c}-\sum_{c j k}(i j \mid c k) \hat{R}_{j k}^{a c}
\end{aligned}
$$

sigma vector elements for singlet excitations, while for the triplet case the sigma vector reads as

$$
\begin{aligned}
\sigma_{i a}= & -\sum_{j b}(i j \mid a b) r_{j}^{b}+\left(\varepsilon_{a}-\varepsilon_{i}\right) r_{i}^{a}+\frac{1}{2} \sum_{k j b c}(j c \mid k b) r_{k}^{c} t_{j i}^{a b}+\frac{1}{2} \sum_{k j b c}(j a \mid i b) r_{k}^{c} t_{j k}^{c b}+\frac{1}{2} \sum_{k j b c}(i b \mid c k)\left(2 t_{j k}^{b c}-t_{k j}^{b c}\right) r_{j}^{a} \\
& +\frac{1}{2} \sum_{k j b c}(j b \mid c k)\left(2 t_{i k}^{b c}-t_{k i}^{b c}\right) r_{j}^{a}-\frac{1}{2} \sum_{k j b c}(j b \mid c k)\left(2 t_{j k}^{a c}-t_{k j}^{a c}\right) r_{i}^{b}-\frac{1}{2} \sum_{k j b c}(j a \mid c k)\left(2 t_{j k}^{b c}-t_{k j}^{b c}\right) r_{i}^{b}+\sum_{b k c}(a b \mid c k) \hat{R}_{i k}^{b c}-\sum_{c j k}(i j \mid c k) \hat{R}_{j k}^{a c}
\end{aligned}
$$

The required $\hat{R}_{\mu_{2}}$ intermediates, having different expressions for the two different kinds of spin multiplicity, are defined in step 4 of the algorithm in Table I and are obtained using the

$$
R_{\mu_{2}}=-\sum_{v_{1}} \frac{A_{\mu_{2} v_{1}} r_{v_{1}}}{\varepsilon_{\mu_{2}}-\omega_{\mathrm{ADC}(2)}}
$$

amplitudes. The additional advantage of solving the rearranged pseudo-eigenvalue problem of Eq. (7) is that the $R_{\mu_{2}}$ amplitudes can be computed "on-the-fly" and their storage is not required.

Our implementation follows the ideas of Hättig and Weigend presented for their density-fitting LR-CC2 algo- rithm. ${ }^{19}$ The main difference is that our working equations are written in the MO basis instead of the atomic orbital (AO) representation of Ref. 19 in order to efficiently utilize the reduced number of orbitals in the compressed NO basis. In the DF approach, the four-center two-electron integrals are approximated in a product form as

$$
(p q \mid r s)=\sum_{Q} J_{p q}^{Q} J_{r s}^{Q}
$$

where capital indices, such as $Q$, denote the functions of the auxiliary basis set and the $\mathbf{J}$ quantities are built from two- and three-center two-electron integrals, $(P \mid Q)$ and $(p q \mid P)$, 
TABLE I. Algorithm and working equations for calculating the sigma vector for singlet and triplet excitations.

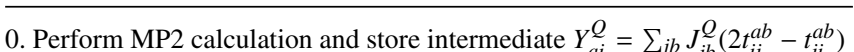

1. Calculate intermediates $X^{Q}, X_{i j}^{Q}$, and $X_{i a}^{Q}$

$$
X^{Q}=2 \sum_{i a} J_{i a}^{Q} r_{i}^{a} \quad X_{i j}^{Q}=\sum_{a} J_{i a}^{Q} r_{j}^{a} \quad X_{i a}^{Q}=\sum_{b} r_{i}^{b} J_{b a}^{Q}
$$

2. Add purely $r_{i}^{a}$-dependent contributions to $\sigma_{i a}$ and construct $\bar{F}_{i a}$ if singlet: $\sigma_{i a} \leftarrow \sum_{Q} J_{i a}^{Q} X^{Q}$

$$
\begin{array}{r}
\quad \bar{F}_{i a}=-\sum_{j Q} J_{j a}^{Q} X_{i j}^{Q}+\sigma_{i a} \\
\text { if triplet: } \bar{F}_{i a}=\sum_{j Q} J_{j a}^{Q} X_{i j}^{Q} \\
\sigma_{i a} \leftarrow-\sum_{j Q} J_{i j}^{Q} X_{j a}^{Q}+\left(\varepsilon_{a}-\varepsilon_{i}\right) r_{i}^{a}
\end{array}
$$

3. Compute $\hat{t}_{i j}^{a b}$, add corresponding contributions to $\sigma_{i a}$

$$
(i a \mid j b)=\sum_{Q} J_{i a}^{Q} J_{j b}^{Q}
$$

if singlet: $\left.\hat{t}_{i j}^{a b}=[2(i a \mid j b)-(j a \mid i b)] / D_{i j}^{a b}\right]$

if triplet: $\hat{t}_{i j}^{a b}=(j a \mid i b) / D_{i j}^{a b}$

$\sigma_{i a} \leftarrow \frac{1}{2} \sum_{j b} \hat{t}_{i j}^{a b} \bar{F}_{j b}$

if singlet: $\sigma_{i a} \leftarrow \frac{1}{2} \sum_{j b} \sum_{k c}[2(i a \mid j b)-(j a \mid i b)] r_{k}^{c} \hat{t}_{j k}^{b c}$

if triplet: $\sigma_{i a} \leftarrow \frac{1}{2} \sum_{j b} \sum_{k c}(j a \mid i b) r_{k}^{c} \hat{t}_{j k}^{b c}$

4. Construct the $\bar{J}_{i a}^{Q}$ list, build intermediate $\hat{R}_{i j}^{a b}$

$\bar{J}_{i a}^{Q}=X_{i a}^{Q}-\sum_{j} J_{i j}^{Q} r_{j}^{a}$

$(\overline{i a} \mid j b)=\sum_{Q} \bar{J}_{i a}^{Q} J_{j b}^{Q} \quad(i a \mid \overline{j b})=\sum_{Q} J_{i a}^{Q} \bar{J}_{j b}^{Q}$

if singlet: $\hat{R}_{i j}^{a b}=[2(\overline{i a} \mid j b)-(\overline{j a} \mid i b)+2(i a \mid \overline{j b})-(j a \mid \overline{i b})] /\left(D_{i j}^{a b}+\omega_{\mathrm{ADC}(2)}\right)$

$$
=\left(2 R_{i j}^{a b}-R_{j i}^{a b}\right) /\left(D_{i j}^{a b}+\omega_{\mathrm{ADC}(2)}\right)
$$

if triplet: $\hat{R}_{i j}^{a b}=[2(\overline{i a} \mid j b)-(\overline{j a} \mid i b)-(j a \mid \overline{i b})] /\left(D_{i j}^{a b}+\omega_{\mathrm{ADC}(2)}\right)$

$\bar{Y}_{i a}^{Q}=\sum_{j b} \hat{R}_{i j}^{a b} J_{j b}^{Q}$

5. Add the remaining contributions to $\sigma_{i a}$

$\sigma_{i a} \leftarrow \sum_{Q b} J_{a b}^{Q} \bar{Y}_{i b}^{Q}$

$\sigma_{i a} \leftarrow-\sum_{Q j} J_{i j}^{Q} \bar{Y}_{j a}^{Q}$

$\sigma_{i a} \leftarrow \frac{1}{2} \sum_{j} \sum_{Q b}\left(J_{i b}^{Q} Y_{j b}^{Q}+J_{j b}^{Q} Y_{i b}^{Q}\right) r_{j}^{a}$

$\sigma_{i a} \leftarrow-\frac{1}{2} \sum_{b} \sum_{Q j}\left(J_{j b}^{Q} Y_{j a}^{Q}+J_{j a}^{Q} Y_{j b}^{Q}\right) r_{i}^{b}$

respectively, as

$$
J_{p q}^{Q}=\sum_{P}(p q \mid P)(P \mid Q)^{-1 / 2} .
$$

The steps of the algorithm are given in detail in Table I. Inspecting the algorithm, one finds the construction of intermediates $\hat{t}_{i j}^{a b}$ and $\hat{R}_{i j}^{a b}$ (steps 3 and 4) from the three-center integrals and the contraction of the latter with the $J_{j b}^{Q}$ list (step 4) as the rate-determining steps of the iterative process. The operation count for these steps is proportional to $n_{\text {occ }}^{2} n_{\text {virt }}^{2} n_{\text {aux }}$, where $n_{\text {occ }}\left(n_{\text {virt }}\right)$ denotes the number of occupied (virtual) orbitals and $n_{\text {aux }}$ stands for the number of auxiliary functions. Since the effective Jacobian, and thus $\sigma$ as well, depends on the excitation energy, the non-linear eigenvalue problem cannot be solved simultaneously for all excited states. To solve the eigenvalue equations effectively, a procedure using a modified Davidson algorithm and the direct inversion in the iterative subspace (DIIS) ${ }^{87}$ algorithm was implemented. ${ }^{19,86}$

At the end of the iteration, the converged $\operatorname{ADC}(2)$ wave function is normalized, which is necessary for the evaluation of transition moments. To achieve this in spatial orbital basis, the amplitudes obtained are divided by the normalization constant

$$
c=\sqrt{\sum_{a i b j} R_{i j}^{a b} R_{i j}^{a b}-\frac{1}{2} \sum_{a i b j} R_{i j}^{a b} R_{j i}^{a b}+\sum_{a i} r_{i}^{a} r_{i}^{a} .}
$$

Then the transition density matrix required for the ground to excited state transition moments can be obtained as

$$
\begin{aligned}
\rho_{p q}= & \left\langle\Psi^{\mathrm{MP} 1}\left|p^{+} q^{-}\right| \Psi^{\mathrm{ADC}(2)}\right\rangle \\
= & \left\langle 0\left|\left(1+T_{2}^{\dagger}\right) p^{+} q^{-}\left(R_{1}+R_{2}\right)\left(1+T_{2}\right)\right| 0\right\rangle \\
= & \left\langle 0\left|p^{+} q^{-} R_{1}\right| 0\right\rangle+\left\langle 0\left|T_{2}^{\dagger} p^{+} q^{-} R_{1}\right| 0\right\rangle \\
& +\left\langle 0\left|T_{2}^{\dagger} p^{+} q^{-} R_{1} T_{2}\right| 0\right\rangle+\left\langle 0\left|T_{2}^{\dagger} p^{+} q^{-} R_{2}\right| 0\right\rangle .
\end{aligned}
$$

This expression is often simplified ${ }^{88,89}$ by discarding disconnected contributions and by neglecting the higher than fifth-power scaling second-order terms. It can be shown that, analogous to LR-CC2, the resulting $\mathrm{ADC}(2)$ density matrix is consistent with the LR-CC theory and correct up to the first order. Our working equations for the transition density matrix in the spatial orbital basis are given for its various blocks by

$$
\begin{gathered}
\rho_{a b}=\sum_{i j c} R_{i j}^{a c}\left(2 t_{i j}^{b c}-t_{j i}^{b c}\right), \\
\rho_{i j}=-\sum_{a b k} R_{i k}^{a b}\left(2 t_{j k}^{a b}-t_{k j}^{a b}\right), \\
\rho_{a i}=\sum_{b j} r_{j}^{b}\left(2 t_{i j}^{a b}-t_{j i}^{a b}\right),
\end{gathered}
$$

and

$$
\begin{aligned}
\rho_{i a} & =r_{i}^{a}-\sum_{k} r_{k}^{a} \sum_{c b j} t_{k j}^{c b}\left(2 t_{i j}^{c b}-t_{j i}^{c b}\right)-\sum_{c} r_{i}^{c} \sum_{b j k} t_{k j}^{c b}\left(2 t_{k j}^{a b}-t_{j k}^{a b}\right) \\
& =r_{i}^{a}-\sum_{k} r_{k}^{a} D_{i k}^{\mathrm{MP} 2}-\sum_{c} r_{i}^{c} D_{a c}^{\mathrm{MP} 2}
\end{aligned}
$$

where $D_{i k}^{\mathrm{MP} 2}$ and $D_{a c}^{\mathrm{MP} 2}$ are the elements, respectively, of the occupied-occupied and the virtual-virtual block of the MP2 one-particle density matrix, and $R_{i j}^{a b}$ of Eq. (12) is, in practice, built from the intermediates of step 4 of Table I as

$$
R_{i j}^{a b}=\frac{(\overline{i a} \mid j b)+(i a \mid \overline{j b})}{D_{i j}^{a b}+\omega_{\mathrm{ADC}(2)}} .
$$

\section{B. Construction of the reduced subspace}

We have shown previously that, while preserving the intrinsic accuracy of the LR-CC2 excitation energies, the dimension of the virtual subspace can be significantly reduced by discarding NOs with low occupation numbers. On the other hand, the analogous frozen occupied NO approximation was found to be far less efficient for LR-CC $2 .{ }^{86}$ We experienced similar trends in the case of $\operatorname{ADC}(2)$, therefore we restrict the present discussion to the application of frozen virtual NOs. If they are of interest, the working equations required for the frozen occupied NO approximation can be obtained analogously.

First, the virtual-virtual block of the one-particle density matrix is constructed from a lower-level wave function $\Psi$,

$$
D_{a b}=\left\langle\Psi\left|a^{+} b^{-}\right| \Psi\right\rangle \text {. }
$$


The eigenvectors of this matrix are the virtual natural orbitals (VNOs), while its eigenvalues are interpreted as the corresponding occupation numbers of the VNOs. If $\Psi$ is appropriately chosen, the NOs with smaller occupation numbers usually give a smaller contribution to the correlation or excitation energies. Therefore, in the frozen NO approximation, the NOs with occupation numbers below a predefined threshold, $\varepsilon_{\mathrm{VNO}}$, are disregarded. The remaining VNOs will be denoted with a tilde (e.g., $\tilde{a}, \tilde{b}, \ldots$ ), and the integral lists transformed to the VNO basis will also be distinguished with tildes (e.g., $\tilde{\mathbf{J}})$.

We have also demonstrated that the state-specific VNOs obtained from state-averaged density matrices are most suitable to efficiently compute LR-CC2 singlet excitation energies. ${ }^{86}$ The state-averaged density matrix we have introduced is defined as $\mathbf{D}=\left(\mathbf{D}^{\mathrm{MP2}}+\mathbf{D}^{\mathrm{CIS}(\mathrm{D})}\right) / 2$, where $\mathbf{D}^{\mathrm{MP2}}$ and $\mathbf{D}^{\mathrm{CIS}(\mathrm{D})}$ denote the density matrices obtained from the MP1 and the configuration interaction singles with perturbative doubles [CIS(D)] wave functions. The one-particle MP2 density matrix in a spatial orbital basis can be expressed in the form of

$$
D_{a b}^{\mathrm{MP} 2}=\sum_{i j c}\left(2 t_{i j}^{c a} t_{i j}^{c b}-t_{i j}^{c a} t_{i j}^{b c}\right) .
$$

The CIS(D) density matrix, $\mathbf{D}^{\mathrm{CIS}(\mathrm{D})}$, is obtained as the sum of the density matrices derived from the CIS wave function and its second-order perturbative correction (D), $\mathbf{D}^{\mathrm{CIS}(\mathrm{D})}=\mathbf{D}^{\mathrm{CIS}}$ $+\mathbf{D}^{(\mathrm{D})}$. The CIS density matrix expressed in spatial orbitals reads as

$$
D_{a b}^{\mathrm{CIS}}=\sum_{i} c_{i}^{a} c_{i}^{b}
$$

for both the singlet and the triplet states, where $c_{i}^{a}$ is a CIS coefficient. The spin adaptation results in different expressions for matrix $\mathbf{D}^{(\mathrm{D})}$ in the singlet and triplet cases. The expression for a singlet state is practically the same as for the MP2 density, Eq. (23), the only difference is that the MP2 amplitudes are substituted by the CIS(D) doubles coefficients. The latter can be expressed as

$$
c_{i j}^{a b}=\frac{\sum_{c}\left[(a c \mid b j) c_{i}^{c}+(a i \mid b c) c_{j}^{c}\right]-\sum_{k}\left[(k j \mid a i) c_{k}^{b}+(k i \mid b j) c_{k}^{a}\right]}{D_{i j}^{a b}+\omega_{\mathrm{CIS}}},
$$

where $\omega_{\text {CIS }}$ stands for the CIS excitation energy. ${ }^{90,91}$ For an excited state of triplet multiplicity, the virtual-virtual block of matrix $\mathbf{D}^{(\mathrm{D})}$ is given as

$$
D_{a b}^{(\mathrm{D})}=\sum_{i j c}\left(\bar{c}_{i j}^{c a} \bar{c}_{i j}^{c b}+c_{i j}^{c a} c_{i j}^{c b}-c_{i j}^{c a} c_{i j}^{b c}\right),
$$

where the $c_{i j}^{a b}$ coefficients are still defined by Eq. (25), but they are built using the triplet CIS coefficients and excitation energy. Furthermore, $\bar{c}_{i j}^{a b}$ denotes the triplet-coupled CIS(D) doubles coefficient, which is evaluated as

$$
\bar{c}_{i j}^{a b}=\frac{\sum_{c}\left[(a c \mid b j) c_{i}^{c}-(a i \mid b c) c_{j}^{c}\right]+\sum_{k}\left[(k j \mid a i) c_{k}^{b}-(k i \mid b j) c_{k}^{a}\right]}{D_{i j}^{a b}+\omega_{\mathrm{CIS}}} .
$$

The algorithms and the working equations for the computation of the singlet density matrix were published previously. ${ }^{86}$ The analogous steps and expressions for the triplet case are
TABLE II. Working equations for the evaluation of the triplet CIS(D) density matrix.

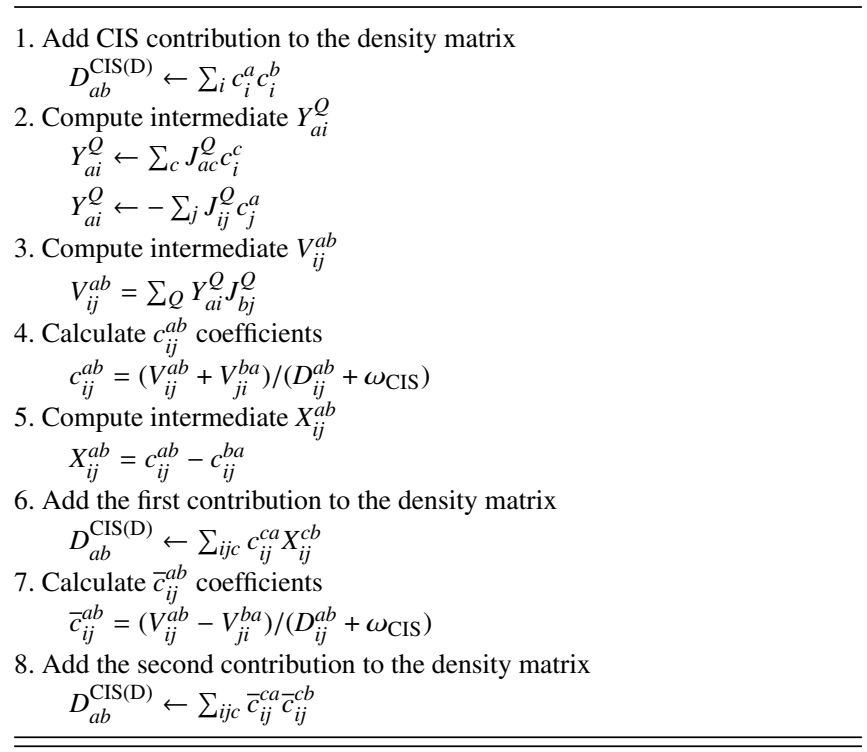

given in detail in Table II. Note that the one-particle MP2 density matrix is only constructed once, for the ground state. On the contrary, the CIS(D) wave function and hence the CIS(D) density matrix have to be evaluated for each excited state. The rate-determining step of the density matrix calculation, for both MP2 and CIS(D), is the assembly of the four-index integral list of intermediate $\mathbf{V}$ (step 3), which is a fifth-power scaling operation with costs proportional to $n_{\mathrm{occ}}^{2} n_{\mathrm{virt}}^{2} n_{\text {aux }}$. The algorithm contains two additional fifth-power scaling operations (steps 6 and 8 ) with computational expenses proportional to $n_{\text {occ }}^{2} n_{\text {virt }}^{3}$, while there is only one such term in the case of MP2 or singlet CIS(D).

In our previous LR-CC2 study, we have also introduced a simplification to the MP2 and (D) density matrices, with which the NO approximation works similarly well. Here we again take advantage of the neglect of the exchange-like terms from the corresponding density matrix expressions. With that approximation Eqs. (23) and (26) read, respectively, as

$$
D_{a b}^{\mathrm{MP} 2}=\sum_{i j c} 2 t_{i j}^{c a} t_{i j}^{c b}
$$

and

$$
D_{a b}^{(\mathrm{D})}=\sum_{i j c}\left(\bar{c}_{i j}^{c a} \bar{c}_{i j}^{c b}+c_{i j}^{c a} c_{i j}^{c b}\right) .
$$

One benefit of this simplification is that the evaluation of intermediate $X_{i j}^{a b}$ (step 5) can be omitted. In addition, the corresponding contribution to the density matrix can be obtained more efficiently via the multiplication of the CIS(D) doubles coefficient matrix with its transpose (step 6). On top of that, the implementation of the algorithm becomes significantly easier even if the entire integral lists or intermediates cannot be kept in the memory.

As expected, the VNOs constructed as described above are only ideal for ADC(2) calculations if CIS(D) is a good approximation to $\operatorname{ADC}(2)$. The prerequisite for this is that the CIS method provides a qualitatively good description of the excited state. If the coefficients of double excitations are not stored, a 
good measure for the quality of the CIS and CIS(D) approximations is the overlap of the CIS and the single excitation part of the final $\operatorname{ADC}(2)$ wave functions. Our numerical experience shows that, if this overlap is relatively small, the $\operatorname{ADC}(2)$ calculations performed in the reduced VNO space may yield inadequate excitation energies and transition moments. In these cases, we found that the dominant excitations of the ADC(2) wave function obtained in the reduced space correspond to those of the CIS wave function, and particular excitations that have considerable weight in the canonical ADC(2) are missing. This problem cannot be resolved by tightening the $\varepsilon_{\mathrm{VNO}}$ threshold as the occupancy of the VNOs which do not noticeably contribute to the CIS wave function is rather low. To overcome this problem, we found the following approach to be useful.

In practice, for a particular excited state, the orbital energies of the canonical virtual orbitals contributing to the wave function are relatively close to each other. Thus, we can suppose that all the important orbitals will be included in the reduced space if it is augmented with the canonical virtual orbitals which are close in energy to the virtuals significantly contributing to the CIS wave function. For the selection of such orbitals, two further thresholds were introduced. First, those canonical virtual orbitals are chosen for which at least one CIS coefficient of an excitation involving them is greater than threshold $\varepsilon_{\mathrm{CIS}}$. Second, all those canonical virtual orbitals are selected whose orbital energy is in a given interval. The lower limit of the interval is the orbital energy of the lowest MO selected in the first step minus threshold $\varepsilon_{E}$, while its upper limit is the orbital energy of the highest chosen MO plus $\varepsilon_{E}$. The orbitals of the reduced VNO space are projected out of the selected canonical virtual orbitals, and the remaining orbitals are orthonormalized employing canonical orthogonalization dropping the eigenvectors of the overlap matrix with eigenvalues lower than $10^{-7}$. The resulting orthonormal MOs are added to the reduced VNO space, and the orbitals of the extended space are canonicalized. In practice, it means that the Fock-matrix is transformed to the extended MO space, and the matrix is diagonalized. The resulting pseudo-canonical orbitals and the corresponding orbital energies will be used in the subsequent calculations. For the sake of simplicity, from now on, this pseudo-canonical basis will be referred to as the VNO basis.

So far an accurate lower-rank approximation to the MP2 and $\mathrm{ADC}(2)$ wave functions has been introduced by defining the corresponding state-specific VNO basis. Analogously, the auxiliary basis can also be compressed by finding an appropriate basis transformation. It can be shown that the truncated singular vector basis of $\mathbf{J}$ gives the most efficient approximation to $\mathbf{J}$ itself and hence to the corresponding assembled four-center integrals. ${ }^{85}$ In practice, it is more economical to obtain the singular vectors as the eigenvalues of matrix $\mathbf{W}$ with elements

$$
W_{P Q}=\sum_{p q} J_{p q}^{P} J_{p q}^{Q}
$$

Similar to the NO approach, the eigenvectors, the so-called NAFs, with eigenvalues below a predefined threshold, $\varepsilon_{\mathrm{NAF}}$, are discarded in the compressed representation of $\mathbf{J}$.
In the following, we will employ two different sets of NAFs. First, as in our previous LR-CC2 study, we construct NAFs to compress the representation of the integrals in the NO basis. Here we build $\mathbf{W}$ of Eq. (30) using $\tilde{\mathbf{J}}$, the integral list transformed to the VNO basis. These NAFs will be referred to as restricted NO space NAFs or RS-NAFs for short. The RS-NAFs are suitable to reduce, for instance, the cost of certain assembly and $\sigma$-vector construction steps of the $\operatorname{ADC}(2)$ calculation (see, e.g., Table I). Another option is to utilize the NAF approximation right at the beginning, as soon as the three-center integrals are transformed to the canonical HF MO basis. These NAFs will be called the complete MO space NAFs, or shortly, CS-NAFs, and the corresponding $\mathbf{J}$ integrals will be denoted hereafter by $\hat{\mathbf{J}}$. We note here that, naturally, the CS-NAF basis is usually significantly larger than the RSNAF basis but still represents a compressed, system-specific auxiliary basis with which the expenses of the CIS calculation and density matrix evaluation steps can be cut roughly in half.

\section{General algorithm}

In this section, we briefly collect all the steps required for our reduced-cost $\operatorname{ADC}(2)$ algorithm. We introduce here two branches for test purposes in order to assess the accuracy of the CS-NAF approximation, which was not present in our previous study ${ }^{86}$ Algorithm 1 is basically the generalization of our VNO and (RS-)NAF approximation-based LR-CC2 scheme apart from the step that the VNOs selected using the $\varepsilon_{\mathrm{VNO}}$ threshold are supplemented with the close-lying canonical orbitals. This is extended in Algorithm 2 by the introduction of CS-NAFs as well (see step 2 below). Our general algorithm is as follows.

0. Solve HF equations

1. Construct the canonical integral list $\mathbf{J}$

2. If Algorithm 2: Calculate matrix $\mathbf{W}$ using three-center integral list $\mathbf{J}$ [Eq. (30)], and transform the auxiliary index of $\mathbf{J}$ to the CS-NAF basis to obtain $\hat{\mathbf{J}}$

3. Solve CIS equations for all the excited states simultaneously using either $\mathbf{J}$ (Algorithm 1) or $\hat{\mathbf{J}}$ (Algorithm 2)

4. Loop over excited states

4.a. Calculate the state-averaged one-particle density matrix D (Table II) using either $\mathbf{J}$ (Algorithm 1) or $\hat{\mathbf{J}}$ (Algorithm 2), and construct the truncated virtual space. Transform the MO indices of $\mathbf{J}$ or $\hat{\mathbf{J}}$ to the VNO basis to obtain $\tilde{\mathbf{J}}$.

4.b. Calculate matrix $\mathbf{W}$ using three-index integrals $\tilde{\mathbf{J}}$ [Eq. (30)], and transform the auxiliary index of $\tilde{\mathbf{J}}$ to the RS-NAF basis $(\overline{\mathbf{J}})$. Write integral list $\overline{\mathbf{J}}$ to disk.

End loop

5. Loop over excited states

5.a. Retrieve integral list $\overline{\mathbf{J}}$ from disk

5.b. Perform MP2 calculation and solve ADC(2) equations (Table I)

End loop

We would like to point out that the CS-NAF approximation affects the accuracy of all the quantities computed 
subsequently, such as density matrices, VNOs, and RS-NAFs. Besides that it is not guaranteed that the truncated RS-NAF basis of Algorithm 1 is a subset of the truncated CS-NAF basis even if the VNOs of the two algorithms can be considered the same in the case of a highly accurate CS-NAF approximation. In other words, if both NAF approximations are employed, contributions to the retained RS-NAFs coming from the dropped CS-NAFs are present in Algorithm 1, but they are lost in Algorithm 2. Both of these error sources will be extensively investigated in Sec. III.

\section{RESULTS}

\section{A. Computational details}

The presented reduced-cost $\operatorname{ADC}(2)$ algorithm has been implemented in the Mrcc suite of quantum chemical programs and is available in the current release of the package. ${ }^{92}$

For the benchmark calculations, Dunning's correlation consistent basis sets augmented with diffuse functions (aug-ccpVXZ, where $X=\mathrm{D}, \mathrm{T}, \mathrm{Q}$ ) were used, ${ }^{93-95}$ and the corresponding auxiliary bases developed by Weigend et al. were employed in both the $\mathrm{HF}$ and the excited-state calculations. ${ }^{96-98}$ In the CIS and $\operatorname{ADC}(2)$ calculations, the core MOs were kept frozen.

To quantify the errors originating from our approximations, a test set of small molecules was employed containing examples for all important types of excitation. ${ }^{86}$ Valence excitations $\left(n \rightarrow \pi^{*}, \pi \rightarrow \pi^{*}\right.$, and $\left.\sigma \rightarrow \pi^{*}\right)$ are present among the transitions taken from the benchmark set of Thiel and co-workers. ${ }^{99,100}$ We have also added a couple of the Rydberg excited states of these systems to our test set as well as the CT excitations of an ethylene-tetrafluoroethylene system
(10 A separation, taken from the work of Dreuw et al. ${ }^{101}$ ). We have also assessed the accuracy of our approach on a more realistic set of systems containing seven medium-sized, frequently studied molecules. ${ }^{86,102-110}$ Additional calculations were performed for even larger systems to demonstrate the efficiency of our implementation. For this purpose, molecules were taken from the studies of Grimme ${ }^{103}$ and Schütz and co-workers. ${ }^{65-69}$ The systems included in the three sets are collected in Table III, while their structures are shown in the supplementary material.

The reported computation times are wall-clock times determined on a machine equipped with 128 GB of main memory and a 6-core 3.5 GHz Intel Xeon E5-1650 processor.

\section{B. Small molecules}

First, we study the accuracy of our approximations on the example of the test set containing the smaller molecules. We analyze the accuracy of the singlet and triplet excitation energies as well as of the oscillator strengths corresponding to singlet transitions using the aug-cc-pVTZ basis set, which is probably the most relevant from the practical point of view; double- and quadruple- $\zeta$-quality basis sets will be considered later. In order to find reliable default values for the VNO and NAF truncation thresholds, we rely on the results of our extensive convergence tests for $\varepsilon_{\mathrm{VNO}}$ and $\varepsilon_{\mathrm{NAF}}$ that we obtained for our related reduced-cost LR-CC2 scheme. $^{86}$ We found, with the default values of $\varepsilon_{\mathrm{VNO}}=7.5 \times 10^{-5}$ and $\varepsilon_{\mathrm{NAF}}=0.1$ a.u., the mean absolute error (MAE) of the singlet LR-CC2 excitation energies to be about $0.02 \mathrm{eV}$, which is an order of magnitude smaller than the intrinsic error of LR-CC2. ${ }^{111,112}$ Considering the close relation of the formulation of $\mathrm{ADC}(2)$ and LR-CC $2,{ }^{113}$ their comparable numerical

TABLE III. The size of the systems studied and the number of the basis functions in the basis sets considered.

\begin{tabular}{|c|c|c|c|c|}
\hline \multirow[b]{2}{*}{ Molecule } & \multirow[b]{2}{*}{ Number of atoms } & \multicolumn{3}{|c|}{ Number of basis functions } \\
\hline & & aug-cc-pVDZ & aug-cc-pVTZ & aug-cc-pVQZ \\
\hline Formaldehyde ${ }^{99,100}$ & 4 & 64 & 138 & 252 \\
\hline Formamide 99,100 & 6 & 96 & 207 & 378 \\
\hline Acetamide $^{99,100}$ & 9 & 137 & 299 & 550 \\
\hline Acetone $\mathrm{e}^{99,100}$ & 10 & 146 & 322 & 596 \\
\hline Butadiene 99,100 & 10 & 146 & 322 & 596 \\
\hline Benzene $^{99,100}$ & 12 & 192 & 414 & 756 \\
\hline Ethylene-tetrafluoroethylene ${ }^{101}$ & 12 & 220 & 460 & 824 \\
\hline Hydrazone dye ${ }^{105}$ & 21 & & 828 & \\
\hline Diphenylamine $^{105}$ & 21 & & 851 & \\
\hline Azobenzene $e^{105}$ & 24 & & 874 & \\
\hline $6,6^{\prime}$-difluoro-indigo ${ }^{102,103}$ & 28 & & 1150 & \\
\hline Bithiophene derivative ${ }^{102,103}$ & 29 & & 1135 & \\
\hline$N$-methyl-2,3-benzocarbazole ${ }^{102,103}$ & 31 & & 1127 & \\
\hline Flavone derivative $\mathrm{e}^{109,110}$ & 36 & & 1311 & \\
\hline $\operatorname{Flv}(a)^{66}$ & 51 & & 2001 & \\
\hline $\operatorname{Dyad}^{65,67,68}$ & 53 & & 2051 & \\
\hline Bisimide derivative $\mathrm{e}^{102,103}$ & 60 & & 2346 & \\
\hline $\operatorname{Flv}(b)^{66}$ & 78 & & 2829 & \\
\hline D21L6 68,69 & 98 & & 3412 & \\
\hline
\end{tabular}


performance, ${ }^{36,64,108,114-116}$ and that our present scheme (especially Algorithm 1) employs similar considerations as our LR-CC2 method, the above default thresholds may seem appropriate for $\mathrm{ADC}(2)$ as well. However, the newly introduced elements of the algorithm require careful consideration. Accordingly, extensive benchmark calculations were carried out to study the size of the errors as a function of the various thresholds. The details can be found in the supplementary material. On the basis of our numerical experience, the VNO and NAF thresholds have not been changed, while we propose $\varepsilon_{\mathrm{CIS}}=0.35$ and $\varepsilon_{E}=0.15$ a.u. as the default thresholds for the virtual space completion. The errors of the approximate $\mathrm{ADC}(2)$ scheme for singlet excitation energies and the corresponding oscillator strengths $(f)$ with respect to canonical ADC(2) are presented in Table IV using the default thresholds for both the VNO and NAF truncations. Looking at the results obtained with Algorithm 1, the MAE for singlet excitation energies in the small-molecule test set is $0.019 \mathrm{eV}$, while the maximum absolute error (MAX) is $0.040 \mathrm{eV}$. This accuracy is achieved by keeping only a fraction of the functions in the transformed bases, precisely, $52.6 \%$ of the VNOs and $81.6 \%$ of the RS-NAFs are dropped on the average. These mean values are quite representative; the fluctuation of the ratio of dropped VNOs and RS-NAFs for this test set is below $10 \%$ and 5\%, respectively. The MAE of the excitation energies is less than that reported for singlet LR-CC2 excitations; however, the number of the VNOs and RS-NAFs is slightly larger ${ }^{86}$ since the originally selected VNOs are augmented with the corresponding canonical orbitals. The errors for the corresponding
$\mathrm{ADC}(2)$ oscillator strengths are also highly acceptable being lower than 0.001 a.u. in average and below 0.005 a.u. in every case. If we compare the accuracy of Algorithm 2 (i.e., when CS-NAFs are also employed at the CIS iterations as well as at the VNO and RS-NAF construction steps) to that of Algorithm 1 , we find its numerical performance just as good. There are only $2 \mathrm{meV}$ and $1 \mathrm{meV}$ differences in their MAE and MAX values, while the oscillator strengths are similarly accurate. This is explained by the fact that, except for a difference of less than $0.5 \%$, the same portion of the VNOs and RS-NAFs is discarded by the two algorithms. The benefit of using Algorithm 2 over Algorithm 1 is that about $60 \%$ of the CS-NAFs can also be dropped without introducing any significant inaccuracy on top of that already present with Algorithm 1. Consequently, most of the steps preceding the $\mathrm{ADC}(2)$ calculation in the reduced VNO/RS-NAF basis can be performed about $60 \%$ faster with Algorithm 2.

To demonstrate the robustness of our approach, we have also studied the triplet excitations of the molecules of the same test set. The triplet excitations considered were selected so that their character and excited-state wave function (in terms of its dominant configurations) will be as close to the singlet excitations included in the test set as possible. Note that the oscillator strength is zero for the spin-forbidden transition from the singlet ground state to the triplet excited state; hence, oscillator strengths are not reported for the triplet excited states. The numerical values for the triplet excitation energies are collected in Table V. The MAE and MAX errors are somewhat larger than the corresponding values obtained for the singlet

TABLE IV. Canonical $\operatorname{ADC}(2)$ singlet excitation energies $(\omega$, in eV), oscillator strength $(f$, in a.u.), the error of excitation energies $(\delta \omega$, in eV) and oscillator strengths ( $\delta f$, in a.u.) with the present approach, and the percentage of VNOs and NAFs dropped using the default thresholds with the aug-cc-pVTZ basis set for small molecules. Oscillator strengths for symmetry-forbidden (s.f.) transitions are not displayed.

\begin{tabular}{|c|c|c|c|c|c|c|c|c|c|c|c|c|c|}
\hline \multirow[b]{2}{*}{ Molecule } & \multirow[b]{2}{*}{ State } & \multirow[b]{2}{*}{ Character } & \multirow[b]{2}{*}{$\omega$} & \multirow[b]{2}{*}{$f$} & \multicolumn{4}{|c|}{ Algorithm 1} & \multicolumn{5}{|c|}{ Algorithm 2} \\
\hline & & & & & $\delta \omega$ & $\delta f$ & $\begin{array}{c}\text { Dropped } \\
\text { VNOs }\end{array}$ & $\begin{array}{l}\text { Dropped } \\
\text { RS-NAFs }\end{array}$ & $\delta \omega$ & $\delta f$ & $\begin{array}{c}\text { Dropped } \\
\text { CS-NAFs }\end{array}$ & $\begin{array}{l}\text { Dropped } \\
\text { VNOs }\end{array}$ & $\begin{array}{l}\text { Dropped } \\
\text { RS-NAFs }\end{array}$ \\
\hline \multirow[t]{3}{*}{ Acetamide } & $\mathrm{S}_{1}$ & $n \rightarrow \pi^{*}$ & 5.356 & 0.000 & 0.005 & 0.000 & 53.7 & 82.3 & 0.006 & 0.000 & 59.3 & 54.1 & 82.6 \\
\hline & $\mathrm{S}_{2}$ & Rydberg & 5.869 & 0.018 & 0.017 & 0.000 & 53.7 & 81.5 & 0.018 & 0.000 & 59.3 & 54.1 & 82.1 \\
\hline & $\mathrm{S}_{3}$ & Rydberg & 6.450 & 0.012 & 0.028 & 0.000 & 51.6 & 81.0 & 0.028 & 0.000 & 59.3 & 51.6 & 81.7 \\
\hline \multirow[t]{3}{*}{ Acetone } & $\mathrm{S}_{1}$ & $n \rightarrow \pi^{*}$ & 4.252 & s.f. & 0.011 & s.f. & 55.9 & 82.9 & 0.017 & s.f. & 60.1 & 56.2 & 83.1 \\
\hline & $\mathrm{S}_{14}$ & $\sigma \rightarrow \pi^{*}$ & 8.959 & 0.000 & 0.009 & 0.000 & 55.6 & 82.9 & 0.012 & 0.000 & 60.1 & 55.6 & 83.0 \\
\hline & $\mathrm{S}_{16}$ & $\pi \rightarrow \pi^{*}$ & 9.102 & 0.000 & 0.011 & -0.005 & 55.6 & 82.9 & 0.015 & -0.005 & 60.1 & 56.2 & 83.4 \\
\hline \multirow[t]{2}{*}{ Benzene } & $\mathrm{S}_{3}$ & $\pi \rightarrow \pi^{*}$ & 6.507 & s.f. & 0.026 & s.f. & 55.5 & 83.7 & 0.026 & s.f. & 61.7 & 55.7 & 83.9 \\
\hline & $\mathrm{S}_{5}$ & $\pi \rightarrow \pi^{*}$ & 7.041 & 0.069 & 0.029 & 0.000 & 56.0 & 83.7 & 0.033 & 0.000 & 61.7 & 57.0 & 84.0 \\
\hline \multirow[t]{2}{*}{ Butadiene } & $\mathrm{S}_{1}$ & $\pi \rightarrow \pi^{*}$ & 6.095 & 0.707 & -0.009 & 0.001 & 54.7 & 83.3 & -0.009 & 0.000 & 61.4 & 55.7 & 83.9 \\
\hline & $\mathrm{S}_{5}$ & $\pi \rightarrow \pi^{*}$ & 7.112 & s.f. & 0.009 & s.f. & 51.5 & 83.4 & 0.008 & s.f. & 61.4 & 52.1 & 83.4 \\
\hline \multirow[t]{3}{*}{ Formaldehyde } & $\mathrm{S}_{1}$ & $n \rightarrow \pi^{*}$ & 3.834 & s.f. & 0.037 & s.f. & 48.5 & 79.3 & 0.036 & s.f. & 59.5 & 50.0 & 80.3 \\
\hline & $\mathrm{S}_{6}$ & $\sigma \rightarrow \pi^{*}$ & 9.030 & 0.001 & 0.016 & 0.000 & 49.2 & 79.6 & 0.020 & 0.000 & 59.5 & 49.2 & 80.3 \\
\hline & $\mathrm{S}_{12}$ & $\pi \rightarrow \pi^{*}$ & 10.557 & 0.038 & 0.026 & 0.000 & 46.9 & 79.9 & 0.025 & 0.000 & 59.5 & 46.9 & 79.9 \\
\hline Formamide & $\mathrm{S}_{3}$ & Rydberg & 6.688 & 0.015 & 0.028 & 0.000 & 49.7 & 79.6 & 0.027 & 0.000 & 59.0 & 49.7 & 80.0 \\
\hline $\mathrm{C}_{2} \mathrm{~F}_{4} \rightarrow \mathrm{C}_{2} \mathrm{H}_{4}$ & $\mathrm{~S}_{17}$ & $\mathrm{CT}$ & 9.577 & 0.000 & 0.040 & 0.000 & 53.0 & 79.6 & 0.041 & 0.000 & 58.1 & 53.0 & 79.8 \\
\hline $\mathrm{C}_{2} \mathrm{H}_{4} \rightarrow \mathrm{C}_{2} \mathrm{~F}_{4}$ & $\mathrm{~S}_{34}$ & $\mathrm{CT}$ & 10.915 & 0.000 & 0.006 & 0.000 & 50.5 & 79.2 & 0.008 & 0.000 & 58.1 & 46.5 & 79.3 \\
\hline Average & & & & & $0.019^{\mathrm{a}}$ & $0.000^{\mathrm{a}}$ & 52.6 & 81.6 & $0.021^{\mathrm{a}}$ & $0.000^{\mathrm{a}}$ & 59.9 & 52.7 & 81.9 \\
\hline Maximum & & & & & $0.040^{\mathrm{b}}$ & $0.005^{\mathrm{b}}$ & 56.0 & 83.7 & $0.041^{b}$ & $0.005^{\mathrm{b}}$ & 61.7 & 57.0 & 84.0 \\
\hline Minimum & & & & & $0.005^{\mathrm{c}}$ & $0.000^{\mathrm{c}}$ & 46.9 & 79.2 & $0.006^{\mathrm{c}}$ & $0.000^{\mathrm{c}}$ & 58.1 & 46.5 & 79.3 \\
\hline
\end{tabular}

a MAE.

${ }^{\mathrm{b}} \mathrm{MAX}$.

${ }^{\mathrm{c}}$ Minimum absolute error (MIN). 
TABLE V. Canonical ADC(2) triplet excitation energies $(\omega$, in $\mathrm{eV})$, the error of excitation energies $(\delta \omega$, in $\mathrm{eV})$ with the present approach, and the percentage of VNOs and NAFs dropped using the default thresholds with the aug-cc-pVTZ basis set for small molecules.

\begin{tabular}{|c|c|c|c|c|c|c|c|c|c|c|}
\hline \multirow[b]{2}{*}{ Molecule } & \multirow[b]{2}{*}{ State } & \multirow[b]{2}{*}{ Character } & \multirow[b]{2}{*}{$\omega$} & \multicolumn{3}{|c|}{ Algorithm 1} & \multicolumn{4}{|c|}{ Algorithm 2} \\
\hline & & & & $\delta \omega$ & $\begin{array}{l}\text { Dropped } \\
\text { VNOs }\end{array}$ & $\begin{array}{l}\text { Dropped } \\
\text { RS-NAFs }\end{array}$ & $\delta \omega$ & $\begin{array}{c}\text { Dropped } \\
\text { CS-NAFs }\end{array}$ & $\begin{array}{l}\text { Dropped } \\
\text { VNOs }\end{array}$ & $\begin{array}{c}\text { Dropped } \\
\text { RS-NAFs }\end{array}$ \\
\hline \multirow[t]{3}{*}{ Acetamide } & $\mathrm{T}_{1}$ & $n \rightarrow \pi^{*}$ & 5.099 & 0.017 & 50.2 & 80.9 & 0.019 & 59.3 & 50.5 & 81.3 \\
\hline & $\mathrm{T}_{2}$ & Rydberg & 5.831 & 0.029 & 55.8 & 81.5 & 0.030 & 59.3 & 55.8 & 82.6 \\
\hline & $\mathrm{T}_{4}$ & Rydberg & 6.298 & 0.035 & 54.1 & 80.9 & 0.034 & 59.3 & 54.1 & 81.7 \\
\hline \multirow[t]{3}{*}{ Acetone } & $\mathrm{T}_{1}$ & $n \rightarrow \pi^{*}$ & 3.887 & 0.025 & 56.2 & 82.7 & 0.026 & 60.1 & 56.2 & 82.9 \\
\hline & $\mathrm{T}_{8}$ & $\sigma \rightarrow \pi^{*}$ & 8.345 & 0.020 & 55.2 & 82.1 & 0.023 & 60.1 & 55.9 & 83.0 \\
\hline & $\mathrm{T}_{9}$ & $\pi \rightarrow \pi^{*}$ & 8.999 & 0.033 & 54.6 & 82.3 & 0.034 & 60.1 & 54.6 & 82.7 \\
\hline \multirow[t]{2}{*}{ Benzene } & $\mathrm{T}_{5}$ & $\pi \rightarrow \pi^{*}$ & 6.468 & 0.033 & 55.5 & 83.6 & 0.032 & 61.7 & 56.0 & 83.7 \\
\hline & $\mathrm{T}_{7}$ & $\pi \rightarrow \pi^{*}$ & 6.986 & 0.038 & 56.5 & 83.4 & 0.038 & 61.7 & 57.0 & 83.8 \\
\hline \multirow[t]{2}{*}{ Butadiene } & $\mathrm{T}_{1}$ & $\pi \rightarrow \pi^{*}$ & 3.434 & 0.027 & 55.7 & 83.3 & 0.027 & 61.4 & 55.7 & 83.3 \\
\hline & $\mathrm{T}_{8}$ & $\pi \rightarrow \pi^{*}$ & 7.483 & 0.013 & 56.4 & 83.6 & 0.013 & 61.4 & 56.7 & 84.0 \\
\hline \multirow[t]{3}{*}{ Formaldehyde } & $\mathrm{T}_{1}$ & $n \rightarrow \pi^{*}$ & 3.375 & 0.048 & 49.2 & 78.9 & 0.047 & 59.5 & 50.0 & 79.6 \\
\hline & $\mathrm{T}_{7}$ & $\sigma \rightarrow \pi^{*}$ & 8.260 & 0.036 & 49.2 & 79.3 & 0.037 & 59.5 & 49.2 & 79.3 \\
\hline & $\mathrm{T}_{14}$ & $\pi \rightarrow \pi^{*}$ & 10.506 & 0.022 & 46.2 & 79.6 & 0.022 & 59.5 & 46.2 & 79.6 \\
\hline Formamide & $\mathrm{T}_{3}$ & Rydberg & 6.159 & 0.037 & 52.8 & 80.9 & 0.038 & 59.0 & 52.8 & 81.1 \\
\hline $\mathrm{C}_{2} \mathrm{~F}_{4} \rightarrow \mathrm{C}_{2} \mathrm{H}_{4}$ & $\mathrm{~T}_{19}$ & $\mathrm{CT}$ & 9.573 & 0.040 & 53.0 & 79.5 & 0.041 & 58.1 & 53.0 & 79.7 \\
\hline $\mathrm{C}_{2} \mathrm{H}_{4} \rightarrow \mathrm{C}_{2} \mathrm{~F}_{4}$ & $\mathrm{~T}_{37}$ & $\mathrm{CT}$ & 10.913 & 0.007 & 50.2 & 78.9 & 0.005 & 58.1 & 50.9 & 79.4 \\
\hline Average & & & & $0.029^{\mathrm{a}}$ & 53.2 & 81.3 & $0.029^{\mathrm{a}}$ & 59.9 & 53.4 & 81.7 \\
\hline Maximum & & & & $0.048^{\mathrm{b}}$ & 56.2 & 83.6 & $0.047^{\mathrm{b}}$ & 61.7 & 57.0 & 84.0 \\
\hline Minimum & & & & $0.007^{\mathrm{c}}$ & 46.2 & 78.9 & $0.005^{\mathrm{c}}$ & 58.1 & 46.2 & 79.3 \\
\hline
\end{tabular}

aAE.

${ }^{\mathrm{b}} \mathrm{MAX}$.

${ }^{\mathrm{c}}$ MIN.

excitations, but both values are more than appropriate. The comparable accuracy can be explained by the fact that the VNO and NAF approximations function almost identically in the two spin cases if the default thresholds are applied. Since we find the CS-NAF approximation similarly accurate for the triplet states as well, we simplify the rest of our numerical analysis by focusing on Algorithm 2 from this point. For the sake of completeness, the corresponding data for Algorithm 1 are presented in the supplementary material.

We also investigated the AO basis-set dependence of our approximations by comparing the results calculated with the aug-cc-pVTZ basis set to those obtained with aug-ccpVDZ and aug-cc-pVQZ. The statistical measures for the corresponding errors are collected in Table VI, while the

TABLE VI. Error measures for the $\operatorname{ADC}(2)$ excitation energies $(\delta \omega$, in $\mathrm{eV})$ and oscillator strengths ( $\delta f$, in a.u.), and the average, maximum, and minimum percentage of VNOs and NAFs dropped using the default thresholds with various basis sets for small molecules.

\begin{tabular}{|c|c|c|c|c|c|c|c|}
\hline & & \multicolumn{2}{|c|}{ aug-cc-pVDZ } & \multicolumn{2}{|c|}{ aug-cc-pVTZ } & \multicolumn{2}{|c|}{ aug-cc-pVQZ } \\
\hline & & Singlet & Triplet & Singlet & Triplet & Singlet & Triplet \\
\hline \multirow[t]{3}{*}{$\delta \omega$} & MAE & 0.045 & 0.039 & 0.021 & 0.029 & 0.064 & 0.080 \\
\hline & MAX & 0.086 & 0.079 & 0.041 & 0.047 & 0.125 & 0.124 \\
\hline & MIN & 0.013 & 0.000 & 0.006 & 0.005 & 0.002 & 0.034 \\
\hline \multirow[t]{3}{*}{$\delta f$} & MAE & 0.001 & $\ldots$ & 0.000 & $\ldots$ & 0.001 & $\ldots$ \\
\hline & MAX & 0.008 & $\ldots$ & 0.005 & $\ldots$ & 0.008 & $\ldots$ \\
\hline & MIN & 0.000 & $\ldots$ & 0.000 & $\cdots$ & 0.000 & $\ldots$ \\
\hline \multirow[t]{3}{*}{ Dropped CS-NAFs } & Avg. & 76.5 & 76.5 & 59.9 & 59.9 & 45.8 & 45.8 \\
\hline & Max. & 77.5 & 77.5 & 61.7 & 61.7 & 47.5 & 47.5 \\
\hline & Min. & 75.9 & 75.9 & 58.1 & 58.1 & 44.7 & 44.7 \\
\hline \multirow[t]{3}{*}{ Dropped VNOs } & Avg. & 18.3 & 17.2 & 52.7 & 53.4 & 73.1 & 73.2 \\
\hline & Max. & 25.1 & 25.1 & 57.0 & 57.0 & 76.2 & 76.8 \\
\hline & Min. & 9.6 & 7.1 & 46.5 & 46.2 & 70.5 & 70.5 \\
\hline \multirow[t]{3}{*}{ Dropped RS-NAFs } & Avg. & 80.5 & 80.3 & 81.9 & 81.7 & 87.8 & 87.9 \\
\hline & Max. & 82.5 & 82.5 & 84.0 & 84.0 & 89.1 & 89.8 \\
\hline & Min. & 77.9 & 77.4 & 79.3 & 79.3 & 86.3 & 86.3 \\
\hline
\end{tabular}


results for the individual molecules are given in Tables SIIISVI of the supplementary material. The MAE and MAX errors of the excitation energies with the aug-cc-pVDZ and aug-cc-pVQZ bases are about 2-3 times larger than the augcc-pVTZ errors. Not surprisingly, the same trend was observed for LR-CC2 in our previous study. ${ }^{86}$ We find this acceptable because the errors are still significantly lower than the intrinsic error of the ADC(2) method. Moreover, usually at least a triple- $\zeta$ basis set augmented with diffuse functions is necessary to bring down the $\mathrm{AO}$ basis-set error to the level comparable to the intrinsic error of $\mathrm{ADC}(2)$ (or LR-CC2). In other words, double- $\zeta$-quality basis sets are less relevant in production level calculations. On the other hand, the use of the quadruple- $\zeta$-quality sets is often not economical or even impossible in practice. Additionally, the error of our approximations for the transition moments is fairly basis set independent.

Considering the basis-set dependence of the ratio of the retained VNOs and NAFs, we again find the similar trend observed previously for LR-CC2. Explicitly, the portion of the neglectable VNOs increases with the basis set size from $18 \%$ through $53 \%$ up to $73 \%$ for aug-cc-pVDZ, aug-cc-pVTZ, and aug-cc-pVQZ, respectively. As expected, larger basis sets can be compressed more when describing the same correlation effect. An opposite trend is present for the CS-NAFs, which is explained by the fact that the ratio of the functions in the $\mathrm{AO}$ and the corresponding auxiliary bases increases with the cardinal number, but the number of the $\mathrm{AO}$ product densities also increases, and a larger portion of the original auxiliary basis is required to be retained for their accurate fitting. Finally, after introducing the system-dependently compressed VNO and CS-NAF spaces, the percentage of the discarded RS-NAFs becomes nearly basis set independent. All in all, the operation count and data size reduction is sizeable with all the basis sets. For instance, the $53 \%$ VNO and the $82 \%$ RS-NAF reduction yield a 25 -fold cut in the operation count in the ratedetermining steps of the $\mathrm{ADC}(2)$ iteration as well as a 25-times smaller three-center integral list with the aug-cc-pVTZ basis. This gain is even larger, almost 120-fold with the aug-cc-pVQZ basis set.

\section{Representative examples}

We have also tested the accuracy of our approximations on larger systems to demonstrate that the errors do not grow with the system size. For that purpose, seven medium-sized dye molecules were collected from the literature (see the middle panel of Table III), which were also useful in realistic applications. Using the default truncation thresholds determined above, we computed the errors of singlet excitation energies and the corresponding oscillator strengths using Algorithm 2 with respect to conventional ADC(2) (see Table VII). Comparing the error measures with those obtained for the smaller molecules (see Table IV), we find a very good agreement. In fact, the MAE (MAX) of singlet excitation energies of the larger systems is even slightly better, being $0.015(0.036) \mathrm{eV}$, than the corresponding value of $0.021(0.041) \mathrm{eV}$ presented in Table IV. We note that the oscillator strengths are more representative from the practical point of view than for the smaller molecules because there is at least one large value (around 0.2 a.u.) for each molecule. However, the errors of the individual oscillator strengths are only moderately larger than those for the smaller test molecules; the quality of the approximate oscillator strengths and the simulated spectra is excellent. The MAE of the oscillator strengths is 0.002 a.u., but two salient errors can be found in the test set. For the $S_{1}$ state of diphenylamine and the $S_{7}$ state of the benzocarbazole derivative, the errors are -0.023 and 0.013 a.u., respectively. In both cases, the CIS wave function is a poor approximation for the corresponding ADC(2) wave function; nevertheless, the improved version of the reduced space construction algorithm results in acceptable errors.

The average portion of the dropped CS-NAFs, VNOs, and RS-NAFs, being about $60 \%, 56 \%$, and $82 \%$, respectively, does not decrease either; hence, similarly good or better speedups are expected in practice for these larger systems.

The above analysis is also performed for the seven lowestlying triplet excitations on the same set of medium-sized dye molecules. If we first compare the errors obtained for the triplet excitations compiled in Table VIII to the corresponding singlet excitation energy errors in Table VII, we find that the triplet MAE is slightly smaller $(0.009$ vs. $0.015 \mathrm{eV})$, while the triplet MAX is a bit worse $(0.042$ vs. $0.036 \mathrm{eV})$, but both values are more than acceptable. A more noticeable difference, 0.009 vs. $0.029 \mathrm{eV}$, appears if the average triplet excitation energy error is compared to that for the smaller test molecules (see Table V). However, this is at least partly explained by the fact that the smaller-molecule test set is less balanced in terms of low- and high-lying excited states. Finally, looking at the portion of the omitted VNOs and NAFs, we find the former to be around 57\% and the latter to be around $82 \%$ in average, which are very close to the corresponding values calculated for the smaller test systems. For the sake of completeness, we also mention that these findings hold not only for the results obtained with Algorithm 2 but with Algorithm 1 as well. The corresponding data for the singlet and triplet excitations are provided in Tables SVII and SVIII of the supplementary material.

So far we have characterized the efficiency of our approximations by reporting the ratio of the omitted VNOs and NAFs and determining the expected operation count reduction at certain steps of the algorithm. An even better measure for that purpose is to compare the actual wall-clock times measured for the conventional and the reduced-cost $\operatorname{ADC}(2)$ variants. The total wall-clock times will be further partitioned into contributions required for the major steps of the computation. For the conventional approach, these parts include the time of the transformation of the three-center integrals from AO to canonical MO basis $\left(t_{\mathbf{J}}\right)$, and the time of the CIS ( $\left.t_{\mathrm{CIS}}\right)$ and the $\mathrm{ADC}(2)\left(t_{\mathrm{ADC}(2)}\right)$ iterations. In the case of the reduced-cost algorithm, the overhead required for the evaluation and diagonalization of the necessary state-averaged density matrices and the $\mathbf{W}$ matrices and the integral transformation to the VNO/NAF bases are accumulated into $t_{\overline{\mathbf{J}}}$. On top of this, in the case of Algorithm 2, the time required for the construction of the CS-NAF basis $\left(t_{\mathrm{CS}-\mathrm{NAF}}\right)$ and the corresponding integral transformation is added to $t_{\mathbf{J}}$ leading to $t_{\hat{\mathbf{J}}}=t_{\mathbf{J}}$ $+t_{\mathrm{CS}-\mathrm{NAF}}$. 
TABLE VII. Canonical ADC(2) singlet excitation energies ( $\omega$, in eV), oscillator strengths ( $f$, in a.u.), the error of excitation energies $(\delta \omega$, in $\mathrm{eV})$ and oscillator strengths $(\delta f$, in a.u.) with the present approach, and the percentage of VNOs and NAFs dropped using the default thresholds with the aug-cc-pVTZ basis set for medium-sized molecules. Oscillator strengths for symmetry-forbidden (s.f.) transitions are not displayed.

\begin{tabular}{|c|c|c|c|c|c|c|c|c|c|}
\hline Molecule & State & Character & $\omega$ & $\delta \omega$ & $f$ & $\delta f$ & $\begin{array}{l}\text { Dropped } \\
\text { CS-NAFs }\end{array}$ & $\begin{array}{l}\text { Dropped } \\
\text { VNOs }\end{array}$ & $\begin{array}{l}\text { Dropped } \\
\text { RS-NAFs }\end{array}$ \\
\hline \multirow[t]{7}{*}{ Hydrazone dye } & $\mathrm{S}_{1}$ & $\pi \rightarrow \pi^{*}$ & 3.475 & -0.002 & 0.111 & -0.001 & 58.7 & 54.1 & 80.4 \\
\hline & $\mathrm{S}_{2}$ & CT & 3.643 & 0.004 & s.f. & s.f. & 58.7 & 55.3 & 80.9 \\
\hline & $\mathrm{S}_{3}$ & $n, \sigma \rightarrow \pi^{*}$ & 3.670 & 0.005 & s.f. & s.f. & 58.7 & 54.5 & 80.7 \\
\hline & $\mathrm{S}_{4}$ & $\pi \rightarrow \pi^{*}$ & 4.073 & -0.007 & 0.459 & -0.004 & 58.7 & 53.4 & 80.3 \\
\hline & $\mathrm{S}_{5}$ & $n, \sigma \rightarrow \pi^{*}$ & 4.268 & 0.006 & 0.000 & 0.000 & 58.7 & 54.5 & 80.7 \\
\hline & $\mathrm{S}_{6}$ & $n, \sigma \rightarrow \pi^{*}$ & 4.287 & 0.006 & 0.001 & 0.000 & 58.7 & 55.2 & 80.9 \\
\hline & $\mathrm{S}_{7}$ & $\pi \rightarrow \pi^{*}$ & 5.007 & -0.026 & 0.050 & -0.006 & 58.7 & 54.0 & 80.5 \\
\hline \multirow[t]{7}{*}{ Azobenzene } & $\mathrm{S}_{1}$ & $n, \sigma \rightarrow \pi^{*}$ & 2.795 & 0.023 & s.f. & s.f. & 61.1 & 58.6 & 83.5 \\
\hline & $\mathrm{S}_{2}$ & $\pi \rightarrow \pi^{*}$ & 4.064 & -0.009 & 0.718 & -0.005 & 61.1 & 57.1 & 83.0 \\
\hline & $\mathrm{S}_{3}$ & $\pi \rightarrow \pi^{*}$ & 4.448 & -0.019 & 0.020 & -0.001 & 61.1 & 56.2 & 82.9 \\
\hline & $\mathrm{S}_{4}$ & $\pi \rightarrow \pi^{*}$ & 4.455 & -0.019 & s.f. & s.f. & 61.1 & 57.4 & 83.0 \\
\hline & $\mathrm{S}_{5}$ & $\pi \rightarrow \pi^{*}$ & 5.182 & -0.024 & s.f. & s.f. & 61.1 & 56.9 & 82.9 \\
\hline & $\mathrm{S}_{6}$ & Rydberg & 6.127 & 0.027 & 0.001 & 0.000 & 61.1 & 56.2 & 83.3 \\
\hline & $\mathrm{S}_{7}$ & Rydberg & 6.315 & 0.028 & s.f. & s.f. & 61.1 & 57.5 & 83.3 \\
\hline \multirow[t]{7}{*}{ Diphenylamine } & $\mathrm{S}_{1}$ & $\pi \rightarrow \pi^{*}$ & 4.335 & -0.011 & 0.104 & -0.023 & 60.8 & 56.9 & 83.5 \\
\hline & $\mathrm{S}_{2}$ & $\pi \rightarrow \pi^{*}$, Ryd & 4.418 & -0.010 & 0.194 & 0.002 & 60.8 & 56.7 & 83.3 \\
\hline & $\mathrm{S}_{3}$ & $\pi \rightarrow \pi^{*}$ & 4.460 & -0.010 & 0.021 & 0.000 & 60.8 & 56.7 & 83.3 \\
\hline & $\mathrm{S}_{4}$ & Rydberg & 4.649 & 0.015 & 0.158 & -0.008 & 60.8 & 56.6 & 83.3 \\
\hline & $\mathrm{S}_{5}$ & Rydberg & 5.045 & 0.027 & 0.049 & -0.004 & 60.8 & 58.3 & 83.8 \\
\hline & $\mathrm{S}_{6}$ & Rydberg & 5.144 & 0.036 & 0.000 & 0.000 & 60.8 & 58.6 & 83.9 \\
\hline & $\mathrm{S}_{7}$ & $\pi \rightarrow \pi^{*}$ & 5.275 & 0.027 & 0.003 & 0.000 & 60.8 & 58.1 & 83.8 \\
\hline \multirow{7}{*}{$\begin{array}{l}6,6^{\prime}- \\
\text { difluoro-indigo }\end{array}$} & $\mathrm{S}_{1}$ & $n, \sigma \rightarrow \pi^{*}$ & 1.965 & 0.006 & 0.000 & 0.000 & 59.9 & 54.7 & 81.0 \\
\hline & $\mathrm{S}_{2}$ & $n, \sigma \rightarrow \pi^{*}$ & 2.479 & 0.004 & s.f. & s.f. & 59.9 & 54.8 & 81.1 \\
\hline & $\mathrm{S}_{3}$ & $\pi \rightarrow \pi^{*}$ & 2.933 & -0.009 & 0.173 & -0.003 & 59.9 & 55.8 & 81.2 \\
\hline & $\mathrm{S}_{4}$ & $\pi \rightarrow \pi^{*}$ & 3.431 & -0.010 & s.f. & s.f. & 59.9 & 54.6 & 81.0 \\
\hline & $\mathrm{S}_{5}$ & $\pi \rightarrow \pi^{*}$ & 3.678 & -0.018 & s.f. & s.f. & 59.9 & 54.4 & 80.9 \\
\hline & $\mathrm{S}_{6}$ & $\pi \rightarrow \pi^{*}$ & 3.697 & -0.017 & 0.140 & 0.000 & 59.9 & 54.4 & 80.9 \\
\hline & $\mathrm{S}_{7}$ & $n, \sigma \rightarrow \pi^{*}$ & 3.748 & -0.003 & s.f. & s.f. & 59.9 & 55.9 & 81.2 \\
\hline \multirow{7}{*}{$\begin{array}{l}\text { Bithiophene } \\
\text { derivative }\end{array}$} & $\mathrm{S}_{1}$ & $\pi \rightarrow \pi^{*}$ & 3.803 & -0.017 & 0.588 & 0.000 & 60.7 & 55.3 & 82.3 \\
\hline & $\mathrm{S}_{2}$ & $\pi \rightarrow \pi^{*}$ & 4.555 & -0.016 & 0.012 & -0.001 & 60.7 & 53.3 & 82.0 \\
\hline & $\mathrm{S}_{3}$ & Rydberg & 4.844 & -0.004 & 0.000 & 0.000 & 60.7 & 55.3 & 82.2 \\
\hline & $\mathrm{S}_{4}$ & $\pi \rightarrow \pi^{*}$ & 4.886 & -0.022 & 0.041 & -0.004 & 60.7 & 55.1 & 82.2 \\
\hline & $\mathrm{S}_{5}$ & Rydberg & 5.034 & 0.005 & 0.001 & 0.000 & 60.7 & 52.4 & 81.9 \\
\hline & $\mathrm{S}_{6}$ & $n, \sigma \rightarrow \pi^{*}$ & 5.497 & -0.003 & 0.001 & 0.000 & 60.7 & 52.4 & 81.9 \\
\hline & $S_{7}$ & $\pi \rightarrow \pi^{*}$ & 5.582 & 0.018 & 0.021 & -0.006 & 60.7 & 55.0 & 82.3 \\
\hline \multirow{7}{*}{$\begin{array}{l}N \text {-methyl-2,3- } \\
\text { benzocarbazole }\end{array}$} & $\mathrm{S}_{1}$ & $\pi \rightarrow \pi^{*}$ & 3.304 & -0.005 & 0.031 & 0.000 & 61.6 & 58.2 & 83.4 \\
\hline & $\mathrm{S}_{2}$ & $\pi \rightarrow \pi^{*}$ & 4.057 & -0.016 & 0.036 & 0.000 & 61.6 & 58.3 & 83.4 \\
\hline & $\mathrm{S}_{3}$ & Rydberg & 4.487 & -0.015 & 0.310 & -0.007 & 61.6 & 59.1 & 83.7 \\
\hline & $\mathrm{S}_{4}$ & Rydberg & 4.516 & 0.033 & 0.007 & 0.000 & 61.6 & 56.9 & 83.2 \\
\hline & $\mathrm{S}_{5}$ & Rydberg & 4.950 & 0.031 & 0.000 & 0.000 & 61.6 & 59.1 & 83.7 \\
\hline & $\mathrm{S}_{6}$ & $\pi \rightarrow \pi^{*}$ & 5.027 & 0.031 & 0.105 & 0.000 & 61.6 & 59.1 & 83.7 \\
\hline & $\mathrm{S}_{7}$ & Rydberg & 5.271 & -0.013 & 0.005 & 0.013 & 61.6 & 57.6 & 83.4 \\
\hline \multirow{7}{*}{$\begin{array}{l}\text { Flavone } \\
\text { derivative }\end{array}$} & $\mathrm{S}_{1}$ & CT & 3.280 & -0.009 & 0.616 & -0.005 & 59.7 & 58.6 & 83.0 \\
\hline & $\mathrm{S}_{2}$ & $\pi \rightarrow \pi^{*}$ & 3.922 & -0.002 & 0.000 & 0.000 & 59.7 & 58.7 & 83.1 \\
\hline & $\mathrm{S}_{3}$ & $n, \sigma \rightarrow \pi^{*}$ & 4.040 & -0.016 & 0.065 & 0.000 & 59.7 & 58.7 & 83.1 \\
\hline & $\mathrm{S}_{4}$ & $\pi \rightarrow \pi^{*}$ & 4.197 & -0.004 & 0.014 & 0.000 & 59.7 & 56.9 & 82.9 \\
\hline & $\mathrm{S}_{5}$ & Rydberg & 4.398 & 0.030 & 0.015 & 0.000 & 59.7 & 59.1 & 83.2 \\
\hline & $\mathrm{S}_{6}$ & $\pi \rightarrow \pi^{*}$ & 4.730 & -0.020 & 0.030 & 0.009 & 59.7 & 58.2 & 82.9 \\
\hline & $\mathrm{S}_{7}$ & Rydberg & 4.869 & 0.024 & 0.001 & 0.000 & 59.7 & 59.0 & 83.2 \\
\hline \multicolumn{4}{|l|}{ Average } & $0.015^{\mathrm{a}}$ & & $0.002^{\mathrm{a}}$ & 60.4 & 56.4 & 82.4 \\
\hline \multicolumn{4}{|l|}{ Maximum } & $0.036^{\mathrm{b}}$ & & $0.023^{\mathrm{b}}$ & 61.6 & 59.1 & 83.9 \\
\hline \multicolumn{4}{|l|}{ Minimum } & $0.002^{\mathrm{c}}$ & & $0.000^{c}$ & 58.7 & 52.4 & 80.3 \\
\hline
\end{tabular}


TABLE VIII. Canonical ADC(2) triplet excitation energies $(\omega$, in $\mathrm{eV})$, the error of excitation energies $(\delta \omega$, in $\mathrm{eV}$ ) with the present approach, and the percentage of VNOs and NAFs dropped using the default thresholds with the aug-cc-pVTZ basis set for small molecules.

\begin{tabular}{|c|c|c|c|c|c|c|c|}
\hline Molecule & State & Character & $\omega$ & $\delta \omega$ & $\begin{array}{l}\text { Dropped } \\
\text { CS-NAFs }\end{array}$ & $\begin{array}{c}\text { Dropped } \\
\text { VNOs }\end{array}$ & $\begin{array}{l}\text { Dropped } \\
\text { RS-NAFs }\end{array}$ \\
\hline \multirow[t]{7}{*}{ Hydrazone dye } & $\mathrm{T}_{1}$ & $\pi \rightarrow \pi^{*}$ & 3.161 & 0.009 & 58.7 & 54.4 & 80.5 \\
\hline & $\mathrm{T}_{2}$ & CT & 3.427 & 0.011 & 58.7 & 55.3 & 80.9 \\
\hline & $\mathrm{T}_{3}$ & $\pi \rightarrow \pi^{*}$ & 3.447 & 0.012 & 58.7 & 54.5 & 80.7 \\
\hline & $\mathrm{T}_{4}$ & $\pi \rightarrow \pi^{*}$ & 3.751 & -0.002 & 58.7 & 54.1 & 80.5 \\
\hline & $\mathrm{T}_{5}$ & $\pi \rightarrow \pi^{*}$ & 3.792 & 0.004 & 58.7 & 54.5 & 80.7 \\
\hline & $\mathrm{T}_{6}$ & CT & 4.189 & 0.004 & 58.7 & 54.8 & 80.7 \\
\hline & $\mathrm{T}_{7}$ & $\pi \rightarrow \pi^{*}$ & 4.279 & 0.013 & 58.7 & 54.5 & 80.7 \\
\hline \multirow[t]{7}{*}{ Azobenzene } & $\mathrm{T}_{1}$ & $n, \sigma \rightarrow \pi^{*}$ & 2.189 & 0.036 & 61.1 & 53.8 & 82.7 \\
\hline & $\mathrm{T}_{2}$ & $\pi \rightarrow \pi^{*}$ & 2.879 & 0.014 & 61.1 & 58.0 & 83.2 \\
\hline & $\mathrm{T}_{3}$ & $\pi \rightarrow \pi^{*}$ & 4.004 & 0.011 & 61.1 & 57.7 & 83.1 \\
\hline & $\mathrm{T}_{4}$ & $\pi \rightarrow \pi^{*}$ & 4.241 & -0.004 & 61.1 & 57.5 & 83.1 \\
\hline & $\mathrm{T}_{5}$ & $\pi \rightarrow \pi^{*}$ & 4.302 & -0.002 & 61.1 & 57.5 & 83.1 \\
\hline & $\mathrm{T}_{6}$ & $\pi \rightarrow \pi^{*}$ & 4.712 & 0.011 & 61.1 & 56.4 & 83.0 \\
\hline & $\mathrm{T}_{7}$ & $\pi \rightarrow \pi^{*}$ & 4.827 & 0.004 & 61.1 & 56.2 & 82.9 \\
\hline \multirow[t]{7}{*}{ Diphenylamine } & $\mathrm{T}_{1}$ & $\pi \rightarrow \pi^{*}$ & 3.634 & 0.012 & 60.8 & 57.6 & 83.4 \\
\hline & $\mathrm{T}_{2}$ & $\pi \rightarrow \pi^{*}$ & 4.103 & 0.008 & 60.8 & 57.1 & 83.3 \\
\hline & $\mathrm{T}_{3}$ & $\pi \rightarrow \pi^{*}$ & 4.110 & 0.003 & 60.8 & 59.2 & 83.7 \\
\hline & $\mathrm{T}_{4}$ & $\pi \rightarrow \pi^{*}$ & 4.263 & 0.009 & 60.8 & 57.2 & 83.4 \\
\hline & $\mathrm{T}_{5}$ & Rydberg & 4.531 & 0.042 & 60.8 & 58.8 & 83.8 \\
\hline & $\mathrm{T}_{6}$ & $\pi \rightarrow \pi^{*}$ & 4.729 & 0.005 & 60.8 & 56.9 & 83.2 \\
\hline & $\mathrm{T}_{7}$ & $\pi \rightarrow \pi^{*}$ & 4.817 & 0.002 & 60.8 & 57.3 & 83.4 \\
\hline \multirow{7}{*}{$\begin{array}{l}6,6^{\prime} \text {-difluoro- } \\
\text { indigo }\end{array}$} & $\mathrm{T}_{1}$ & $\pi \rightarrow \pi^{*}$ & 2.087 & 0.011 & 59.9 & 56.0 & 81.2 \\
\hline & $\mathrm{T}_{2}$ & $\pi \rightarrow \pi^{*}$ & 2.786 & 0.006 & 59.9 & 54.6 & 80.9 \\
\hline & $\mathrm{T}_{3}$ & $\pi \rightarrow \pi^{*}$ & 3.286 & -0.003 & 59.9 & 54.5 & 80.9 \\
\hline & $\mathrm{T}_{4}$ & $\pi \rightarrow \pi^{*}$ & 3.448 & 0.001 & 59.9 & 54.6 & 81.0 \\
\hline & $\mathrm{T}_{5}$ & $\pi \rightarrow \pi^{*}$ & 4.212 & 0.002 & 59.9 & 52.9 & 80.8 \\
\hline & $\mathrm{T}_{6}$ & $\pi \rightarrow \pi^{*}$ & 4.292 & -0.010 & 59.9 & 55.7 & 81.1 \\
\hline & $\mathrm{T}_{7}$ & $\pi \rightarrow \pi^{*}$ & 4.357 & 0.002 & 59.9 & 52.9 & 80.7 \\
\hline \multirow{7}{*}{$\begin{array}{l}\text { Bithiophene } \\
\text { derivative }\end{array}$} & $\mathrm{T}_{1}$ & $\pi \rightarrow \pi^{*}$ & 2.697 & 0.011 & 61.6 & 55.4 & 82.2 \\
\hline & $\mathrm{T}_{2}$ & $\pi \rightarrow \pi^{*}$ & 3.863 & 0.011 & 61.6 & 53.6 & 82.0 \\
\hline & $\mathrm{T}_{3}$ & $\pi \rightarrow \pi^{*}$ & 4.013 & 0.009 & 61.6 & 55.5 & 82.3 \\
\hline & $\mathrm{T}_{4}$ & $\pi \rightarrow \pi^{*}$ & 4.301 & 0.007 & 61.6 & 53.7 & 82.1 \\
\hline & $\mathrm{T}_{5}$ & Rydberg & 4.784 & 0.004 & 61.6 & 55.4 & 81.9 \\
\hline & $\mathrm{T}_{6}$ & $\pi \rightarrow \pi^{*}$ & 5.262 & -0.001 & 61.6 & 52.1 & 82.1 \\
\hline & $\mathrm{T}_{7}$ & $n, \sigma \rightarrow \pi^{*}$ & 5.380 & -0.005 & 61.6 & 55.2 & 82.2 \\
\hline \multirow{7}{*}{$\begin{array}{l}N \text {-methyl-2,3- } \\
\text { benzocarbazole }\end{array}$} & $\mathrm{T}_{1}$ & $\pi \rightarrow \pi^{*}$ & 2.703 & 0.011 & 60.7 & 58.7 & 83.5 \\
\hline & $\mathrm{T}_{2}$ & $\pi \rightarrow \pi^{*}$ & 3.487 & 0.004 & 60.7 & 58.5 & 83.5 \\
\hline & $\mathrm{T}_{3}$ & $\pi \rightarrow \pi^{*}$ & 3.764 & 0.003 & 60.7 & 58.4 & 83.4 \\
\hline & $\mathrm{T}_{4}$ & $\pi \rightarrow \pi^{*}$ & 4.100 & 0.006 & 60.7 & 58.4 & 83.4 \\
\hline & $\mathrm{T}_{5}$ & $\pi \rightarrow \pi^{*}$ & 4.379 & -0.020 & 60.7 & 59.5 & 83.5 \\
\hline & $\mathrm{T}_{6}$ & $\pi \rightarrow \pi^{*}$ & 4.489 & 0.008 & 60.7 & 59.1 & 83.7 \\
\hline & $\mathrm{T}_{7}$ & $\pi \rightarrow \pi^{*}$ & 4.604 & -0.009 & 60.7 & 59.6 & 83.7 \\
\hline \multirow{7}{*}{$\begin{array}{l}\text { Flavone } \\
\text { derivative }\end{array}$} & $\mathrm{T}_{1}$ & CT & 2.585 & 0.010 & 59.7 & 59.0 & 83.1 \\
\hline & $\mathrm{T}_{2}$ & $n, \sigma \rightarrow \pi^{*}$ & 3.537 & 0.002 & 59.7 & 59.0 & 83.1 \\
\hline & $\mathrm{T}_{3}$ & $\pi \rightarrow \pi^{*}$ & 3.940 & 0.011 & 59.7 & 59.0 & 83.1 \\
\hline & $\mathrm{T}_{4}$ & $n, \sigma \rightarrow \pi^{*}$ & 4.007 & 0.004 & 59.7 & 58.8 & 82.9 \\
\hline & $\mathrm{T}_{5}$ & $\pi \rightarrow \pi^{*}$ & 4.277 & -0.008 & 59.7 & 57.1 & 82.8 \\
\hline & $\mathrm{T}_{6}$ & $\pi \rightarrow \pi^{*}$ & 4.650 & 0.001 & 59.7 & 57.2 & 82.9 \\
\hline & $\mathrm{T}_{7}$ & $\pi \rightarrow \pi^{*}$ & 4.738 & 0.035 & 59.7 & 59.0 & 83.1 \\
\hline \multicolumn{4}{|l|}{ Average } & $0.009^{\mathrm{a}}$ & 60.4 & 56.5 & 82.4 \\
\hline \multicolumn{4}{|l|}{ Maximum } & $0.042^{\mathrm{b}}$ & 61.6 & 59.6 & 83.8 \\
\hline \multicolumn{4}{|l|}{ Minimum } & $0.001^{\mathrm{c}}$ & 58.7 & 52.1 & 80.5 \\
\hline
\end{tabular}


TABLE IX. The wall-clock times required for the various steps of the calculations in minutes.

\begin{tabular}{|c|c|c|c|c|c|c|c|c|c|c|}
\hline \multirow[b]{2}{*}{ Molecule } & \multicolumn{4}{|c|}{ Canonical } & \multicolumn{5}{|c|}{ Algorithm 2} & \multirow[b]{2}{*}{ Speedup } \\
\hline & $t_{\mathbf{J}}$ & $t_{\mathrm{CIS}}$ & $t_{\mathrm{ADC}(2)}$ & $t_{\text {Total }}$ & $t_{\hat{\mathbf{J}}}$ & $t_{\mathrm{CIS}}$ & $t_{\overline{\mathbf{J}}}$ & $t_{\mathrm{ADC}(2)}$ & $t_{\text {Total }}$ & \\
\hline Azobenzene & 6.2 & 4.4 & 352.6 & 363.1 & 6.5 & 1.7 & 6.7 & 19.7 & 34.6 & 10.5 \\
\hline Hydrazone dye & 4.9 & 3.8 & 492.8 & 501.5 & 5.1 & 1.5 & 7.6 & 35.9 & 50.1 & 10.0 \\
\hline Diphenylamine & 5.6 & 3.2 & 511.8 & 520.5 & 6.0 & 1.3 & 6.2 & 20.4 & 33.9 & 15.4 \\
\hline Benzocarbazole derivative & 16.9 & 10.2 & 2218.1 & 2245.2 & 18.0 & 4.2 & 20.3 & 97.7 & 140.2 & 16.0 \\
\hline Indigo derivative & 17.3 & 10.9 & 2032.4 & 2060.5 & 18.2 & 4.2 & 31.9 & 125.3 & 179.6 & 11.5 \\
\hline Bithiophene derivative & 16.8 & 13.7 & 2894.5 & 2925.0 & 17.9 & 5.1 & 30.8 & 185.7 & 239.6 & 12.2 \\
\hline Flavone derivative & 29.8 & 19.1 & 4926.7 & 4975.6 & 31.7 & 7.5 & 47.9 & 201.8 & 288.9 & 17.2 \\
\hline Average & & & & & & & & & & 13.3 \\
\hline Maximum & & & & & & & & & & 17.2 \\
\hline Minimum & & & & & & & & & & 10.0 \\
\hline
\end{tabular}

Our wall-clock time measurements for the lowest-lying seven singlet and seven triplet excitation energies and the corresponding singlet transition moments of the seven mediumsized test molecules discussed previously are presented in Table IX for Algorithm 2. The analogous wall-clock times with Algorithm 1 are given in Table SIX of the supplementary material. Most importantly, the average overall speedup factor with Algorithm 2 compared to the conventional algorithm is about 13 using the same hardware. This significant efficiency increase occurs quite reliably considering that the maximum (minimum) overall speedup is 17.2 (10.0). It can be seen that the time required for the CIS iterations as well as for the construction of VNOs and RS-NAFs can be halved compared to Algorithm 1 (cf. Table SIX of the supplementary material), while the additional computation cost needed for the construction of the CS-NAF basis with respect to the evaluation of the canonical integral list $\mathbf{J}$ is only about $5 \%$. Consequently, the total calculation can be performed about $25 \%$ faster (and with considerably reduced storage requirement) via Algorithm 2. We expect similar benefits from the introduction of CS-NAFs in our reduced-cost LR-CC2 method. ${ }^{86}$

To demonstrate the potential of our approach, we determined the excitation energies and oscillator strengths for even larger molecules containing up to 98 atoms using the augcc-pVTZ basis set. Such extensive ADC(2) calculations with more than 3400 AOs are prohibitive or at least highly challenging with the conventional $\mathrm{ADC}(2)$ implementations. The calculated excited-state properties are collected in Table X for Algorithm 2, while Table SX of the supplementary material contains the analogous data for Algorithm 1. Again, the numerical result for the excitation energies and oscillator strengths computed with the two algorithms agree within a few thousandths of an $\mathrm{eV}$ and a.u., respectively. The characters and the energies of the studied singlet excitations match the ones obtained previously with our reduced-cost LR-CC2 method. ${ }^{86}$ The percentage of the dropped VNOs and RS-NAFs lies again in the previously experienced regions at around $60 \%$ and $83 \%$, respectively. Since these five systems cover a sizable range containing 51-98 atoms and 2001-3412 AOs, it seems normal that we observe a slight shift in the ratio of the omitted basis functions when going towards the larger systems. This increase is, however, only about $6 \%$ for the VNOs and $2 \%$ for the RS-NAFs and can at least partially be attributed to the increasing quasi-redundancy in the augmented triple- $\zeta$ $\mathrm{AO}$ basis of these extended molecules. We also present in Table X the measured wall-clock times for the most demanding operations using Algorithm 2. Compared to the results of the measurements performed for the medium-sized systems, these timings are affected by the fact that neither the complete canonical integral list $\mathbf{J}$ nor its CS-NAF compressed $\hat{\mathbf{J}}$ variant fits into the memory. For such cases, we also implemented the out-of-core versions of the necessary operations where the entire integral list is stored on the hard disk, and only blocks of suitable size are processed at a time. The use of the out-of-core algorithms is only required until the integral transformation to the VNO/RS-NAF bases is complete, for the $\mathrm{ADC}(2)$ iterations our scheme benefits from the greatly reduced data sizes in the compressed $\mathrm{MO}$ /auxiliary function spaces.

Looking at the wall-clock times, we can conclude that a single CIS iteration takes somewhat smaller time for the triplet states. This result is not surprising since the calculation of the Coulomb-term is not necessary. However, as it can be inferred from Table II, the construction of the triplet CIS(D) density matrix requires more operations; hence, $t_{\overline{\mathrm{J}}}$ is about $20 \%$ more expensive for the triplet states. An $\mathrm{ADC}(2)$ iteration takes slightly less time for the singlet states although it requires somewhat more operations. However, a couple of rate-determining steps (see in Table I) can be implemented more efficiently for singlet excitations. Compared to the $\mathrm{ADC}(2)$ excitation energy computation, the cost of the transition moments is almost negligible, taking only the time of roughly 1.2 iterations. If we compare the performance of the two algorithms (cf. Table X and Table SX of the supplementary material), we observe a small increase (from 5\% to $20 \%$ ) in the overhead required for the CS-NAF construction. This is explained by the disk I/O demand of the out-of-core CS-NAF algorithm not present previously. This cost increase is, however, amply compensated by the about $50 \%$ percent more economical CIS iteration and VNO as well as RS-NAF construction of Algorithm 2. In principle, the expenses of the $\mathrm{ADC}(2)$ iterations are independent of the choice of the auxiliary basis used for the VNO density matrix construction 
TABLE X. ADC(2) excitation energies ( $\omega$, in eV) and oscillator strengths $(f$, in a.u.) computed with the present approach, the percentage of VNOs and NAFs dropped, and computation times (in min) using the default thresholds with the aug-cc-pVTZ basis set for the largest molecules.

\begin{tabular}{|c|c|c|c|c|c|c|c|c|c|c|c|c|}
\hline Molecule & State & Character & $\omega$ & $f$ & $\begin{array}{l}\text { Dropped } \\
\text { CS-NAFs }\end{array}$ & $\begin{array}{c}\text { Dropped } \\
\text { VNOs }\end{array}$ & $\begin{array}{l}\text { Dropped } \\
\text { RS-NAFs }\end{array}$ & $t_{\hat{\mathbf{J}}}$ & $t_{\mathrm{CIS}}{ }^{\mathrm{a}}$ & $t_{\overline{\mathbf{J}}}^{\mathrm{b}}$ & $t_{\mathrm{ADC}(2)}{ }^{\mathrm{c}}$ & $t_{f}{ }^{\mathrm{d}}$ \\
\hline \multirow[t]{8}{*}{ Flv(a) } & $\mathrm{S}_{1}$ & $\pi \rightarrow \pi^{*}$ & 2.601 & 0.303 & 59.2 & 58.1 & 82.2 & 226.3 & 1.4 & 34.8 & 4.0 & 5.3 \\
\hline & $\mathrm{S}_{2}$ & $\pi \rightarrow \pi^{*}$ & 2.868 & 0.187 & 59.2 & 58.1 & 82.1 & & & 34.8 & 4.1 & 5.1 \\
\hline & $\mathrm{S}_{3}$ & $n, \sigma \rightarrow \pi^{*}$ & 3.309 & 0.001 & 59.2 & 58.4 & 82.2 & & & 34.8 & 3.7 & 5.0 \\
\hline & $\mathrm{S}_{4}$ & $\pi \rightarrow \pi^{*}$ & 3.511 & 0.112 & 59.2 & 58.1 & 82.2 & & & 34.8 & 3.7 & 5.0 \\
\hline & $\mathrm{T}_{1}$ & $\pi \rightarrow \pi^{*}$ & 1.870 & $\ldots$ & 59.2 & 58.3 & 82.2 & & 1.1 & 43.6 & 4.7 & $\ldots$ \\
\hline & $\mathrm{T}_{2}$ & $\pi \rightarrow \pi^{*}$ & 2.367 & $\ldots$ & 59.2 & 58.3 & 82.1 & & & 43.5 & 4.5 & $\ldots$ \\
\hline & $\mathrm{T}_{3}$ & $\mathrm{CT}$ & 3.241 & $\ldots$ & 59.2 & 58.1 & 82.1 & & & 43.5 & 4.7 & $\ldots$ \\
\hline & $\mathrm{T}_{4}$ & $\pi \rightarrow \pi^{*}$ & 3.602 & $\ldots$ & 59.2 & 58.3 & 82.1 & & & 43.4 & 4.5 & $\ldots$ \\
\hline \multirow[t]{8}{*}{ Dyad } & $\mathrm{S}_{1}$ & $\pi \rightarrow \pi^{*}$ & 2.944 & 0.169 & 59.8 & 58.3 & 82.8 & 254.7 & 1.4 & 35.8 & 4.4 & 4.9 \\
\hline & $\mathrm{S}_{2}$ & $\mathrm{CT}$ & 3.314 & 0.001 & 59.8 & 58.6 & 82.9 & & & 35.9 & 4.3 & 4.8 \\
\hline & $\mathrm{S}_{3}$ & $\pi \rightarrow \pi^{*}$ & 3.486 & 0.142 & 59.8 & 56.4 & 82.5 & & & 35.8 & 4.8 & 5.4 \\
\hline & $\mathrm{S}_{4}$ & $\mathrm{CT}$ & 3.790 & 0.045 & 59.8 & 58.1 & 82.8 & & & 35.8 & 4.3 & 4.8 \\
\hline & $\mathrm{T}_{1}$ & $\pi \rightarrow \pi^{*}$ & 2.447 & $\ldots$ & 59.8 & 58.4 & 82.8 & & 1.1 & 45.0 & 4.3 & $\ldots$ \\
\hline & $\mathrm{T}_{2}$ & $\mathrm{CT}$ & 3.010 & $\ldots$ & 59.8 & 56.6 & 82.5 & & & 45.2 & 5.0 & $\ldots$ \\
\hline & $\mathrm{T}_{3}$ & $\mathrm{CT}$ & 3.342 & $\ldots$ & 59.8 & 58.3 & 82.8 & & & 45.3 & 4.6 & $\ldots$ \\
\hline & $\mathrm{T}_{4}$ & $\pi \rightarrow \pi^{*}$ & 3.701 & $\ldots$ & 59.8 & 58.2 & 82.7 & & & 45.1 & 4.5 & $\ldots$ \\
\hline \multirow[t]{8}{*}{ Bisimide derivative } & $\mathrm{S}_{1}$ & $\pi \rightarrow \pi^{*}$ & 2.481 & 0.683 & 58.3 & 59.7 & 82.7 & 375.3 & 7.8 & 71.1 & 8.4 & 9.3 \\
\hline & $\mathrm{S}_{2}$ & $\pi \rightarrow \pi^{*}$ & 3.434 & 0.000 & 58.3 & 59.8 & 82.8 & & & 71.1 & 7.9 & 9.3 \\
\hline & $\mathrm{S}_{3}$ & $\pi \rightarrow \pi^{*}$ & 3.690 & 0.021 & 58.3 & 59.8 & 82.8 & & & 71.1 & 7.9 & 9.3 \\
\hline & $\mathrm{S}_{4}$ & $\pi \rightarrow \pi^{*}$ & 3.782 & 0.000 & 58.3 & 57.3 & 82.5 & & & 71.1 & 8.6 & 9.9 \\
\hline & $\mathrm{T}_{1}$ & $\pi \rightarrow \pi^{*}$ & 1.600 & $\ldots$ & 58.3 & 59.8 & 82.7 & & 7.7 & 87.3 & 8.4 & $\ldots$ \\
\hline & $\mathrm{T}_{2}$ & $\pi \rightarrow \pi^{*}$ & 2.993 & $\ldots$ & 58.3 & 57.6 & 82.5 & & & 87.2 & 9.8 & $\ldots$ \\
\hline & $\mathrm{T}_{3}$ & $\pi \rightarrow \pi^{*}$ & 3.079 & $\ldots$ & 58.3 & 57.5 & 82.5 & & & 87.2 & 9.4 & $\ldots$ \\
\hline & $\mathrm{T}_{4}$ & $\mathrm{CT}$ & 4.267 & $\ldots$ & 58.3 & 60.1 & 82.9 & & & 87.3 & 8.3 & $\ldots$ \\
\hline \multirow[t]{8}{*}{ Flv(b) } & $\mathrm{S}_{1}$ & $\pi \rightarrow \pi^{*}$ & 2.431 & 0.368 & 57.9 & 60.7 & 83.3 & 835.8 & 22.7 & 166.8 & 17.4 & 20.2 \\
\hline & $\mathrm{S}_{2}$ & $\pi \rightarrow \pi^{*}$ & 2.847 & 0.169 & 57.9 & 61.5 & 83.4 & & & 166.8 & 16.4 & 19.1 \\
\hline & $\mathrm{S}_{3}$ & $n, \sigma \rightarrow \pi^{*}$ & 3.219 & 0.148 & 57.9 & 60.8 & 83.3 & & & 166.7 & 16.9 & 19.7 \\
\hline & $\mathrm{S}_{4}$ & $\pi \rightarrow \pi^{*}$ & 3.267 & 0.000 & 57.9 & 61.6 & 83.5 & & & 166.7 & 16.5 & 19.0 \\
\hline & $\mathrm{T}_{1}$ & $\pi \rightarrow \pi^{*}$ & 1.741 & $\ldots$ & 57.9 & 60.9 & 83.3 & & 16.8 & 203.4 & 15.7 & $\ldots$ \\
\hline & $\mathrm{T}_{2}$ & $\pi \rightarrow \pi^{*}$ & 2.353 & $\ldots$ & 57.9 & 61.6 & 83.5 & & & 203.3 & 16.9 & $\ldots$ \\
\hline & $\mathrm{T}_{3}$ & $\pi \rightarrow \pi^{*}$ & 2.727 & $\ldots$ & 57.9 & 61.0 & 83.4 & & & 203.4 & 17.9 & $\ldots$ \\
\hline & $\mathrm{T}_{4}$ & $\pi \rightarrow \pi^{*}$ & 4.078 & $\ldots$ & 57.9 & 61.6 & 83.5 & & & 203.3 & 17.7 & $\ldots$ \\
\hline \multirow[t]{8}{*}{ D21L6 } & $\mathrm{S}_{1}$ & $\mathrm{CT}$ & 2.600 & 1.056 & 59.0 & 61.9 & 84.4 & 1468.3 & 38.0 & 370.6 & 38.6 & 40.3 \\
\hline & $\mathrm{S}_{2}$ & $\pi \rightarrow \pi^{*}$ & 3.368 & 0.109 & 59.0 & 60.6 & 84.2 & & & 370.7 & 39.3 & 42.2 \\
\hline & $\mathrm{S}_{3}$ & Rydberg & 3.464 & 0.061 & 59.0 & 61.6 & 84.3 & & & 370.7 & 37.6 & 41.6 \\
\hline & $\mathrm{S}_{4}$ & Rydberg & 4.044 & 0.019 & 59.0 & 61.5 & 84.3 & & & 370.8 & 37.6 & 41.3 \\
\hline & $\mathrm{T}_{1}$ & CT & 1.972 & $\ldots$ & 59.0 & 62.0 & 84.4 & & 25.2 & 455.5 & 37.1 & $\ldots$ \\
\hline & $\mathrm{T}_{2}$ & $\pi \rightarrow \pi^{*}$ & 2.601 & $\ldots$ & 59.0 & 61.9 & 84.4 & & & 455.7 & 38.0 & $\ldots$ \\
\hline & $\mathrm{T}_{3}$ & $\pi \rightarrow \pi^{*}$ & 3.253 & $\ldots$ & 59.0 & 61.7 & 84.3 & & & 455.6 & 37.9 & $\ldots$ \\
\hline & $\mathrm{T}_{4}$ & $\mathrm{CT}$ & 3.482 & $\ldots$ & 59.0 & 61.9 & 84.3 & & & 455.9 & 37.5 & $\ldots$ \\
\hline Average & & & & & 58.8 & 59.6 & 83.1 & & & & & \\
\hline Maximum & & & & & 59.8 & 62.0 & 84.4 & & & & & \\
\hline Minimum & & & & & 57.9 & 56.4 & 82.1 & & & & & \\
\hline
\end{tabular}

a Average wall-clock time for a CIS iteration using a multi-state algorithm.

${ }^{\mathrm{b}}$ Average wall-clock time for the construction of the $\overline{\mathbf{J}}$ integral list. The calculation of the MP2 density is distributed equally among the excited states.

${ }^{\mathrm{c}}$ Average wall-clock time for an $\mathrm{ADC}(2)$ iteration.

${ }^{\mathrm{d}}$ Wall-clock time required for the oscillator strength calculation.

(i.e., conventional vs. CS-NAF). As expected, in practice, we only see a negligible difference due to the fact that the size of the VNO and RS-NAF bases with Algorithms 1 and 2 are not completely identical but are very similar.

\section{CONCLUSIONS}

An improved version of our virtual natural orbital and natural auxiliary function-based approximation ${ }^{86}$ has been presented, and the approach has been extended to the evaluation of triplet excitation energies and ground to excited state transition moments. In contrast to our previous approach, in the modified scheme the NAF approximation is invoked right at the beginning of the calculation, and the originally selected VNOs are supplemented with canonical orbitals. With the improved algorithm, we have significantly reduced the computation time required for the solution of the CIS equations and the construction of the VNOs and NAFs 
necessary for the correlated excited-state calculations, which were among the rate-determining steps previously. Furthermore, the augmented reduced subspace considerably improves the robustness of the method and the accuracy of the computed properties.

We have derived and implemented the required working equations for the triplet CIS(D) density matrix and set up the infrastructure for the calculation of triplet excitation energies. The efficiency of the approximation has been demonstrated for the ADC(2) method, which is currently one of the most promising low-scaling correlated approaches for excitedstate properties. We have implemented the working equations for $\mathrm{ADC}(2)$ singlet and triplet excitation energies as well as transition moments in the framework of our reduced-cost scheme.

Extensive benchmark calculations have been performed for singlet and triplet excitation energies as well as oscillator strengths for a representative test set including excitations of all the important types. On the basis of the results obtained, it can be stated that the average errors in the singlet and triplet excitation energies $(0.02 \mathrm{eV})$ are an order of magnitude smaller than the error of the ADC(2) method. The maximum absolute error in the computed oscillator strengths does not exceeded 0.025 a.u., while the mean absolute error is 0.002 a.u. The percentage of the neglected VNOs and NAFs seems to be constant independently of the size of the system for any type of excitation. With these approximations, an average speedup of more than an order of magnitude can be achieved for ADC(2) calculations. Our results also demonstrate that, using the new approach, the excited states of extended systems including up to 100 atoms can be routinely studied with reliable basis sets at the $\mathrm{ADC}(2)$ level.

\section{SUPPLEMENTARY MATERIAL}

See supplementary material for molecular structures, excitation energies, and oscillator strengths computed with various algorithms and basis sets.

\section{ACKNOWLEDGMENTS}

The authors are thankful to Professor Andreas Köhn for helpful discussions regarding the implementation of the transition densities. The authors are grateful for the financial support from the National Research, Development, and Innovation Office (NKFIH, Grant No. KKP126451). The work of P.R.N. is supported through the New National Excellence Program of the Ministry of Human Capacities, ID: ÚNKP17-4-BME-55. The computing time granted on the Hungarian HPC Infrastructure at NIIF Institute, Hungary, is gratefully acknowledged.

\footnotetext{
${ }^{1}$ M. E. Casida, "Recent advances in density functional methods," in Computational Chemistry: Reviews of Current Trends, edited by D. P. Chong (World Scientific, Singapore, 1999), Vol. 1.

${ }^{2}$ M. E. Casida and M. Huix-Rotllant, Annu. Rev. Phys. Chem. 63, 287 (2012).

${ }^{3}$ J. Ridley and M. Zerner, Theor. Chim. Acta 32, 111 (1973).

${ }^{4}$ M. C. Zerner, G. H. Loew, R. F. Kirchner, and U. T. Mueller-Westerhof,

J. Am. Chem. Soc. 102, 589 (1980)

${ }^{5}$ W. Weber and W. Thiel, Theor. Chem. Acc. 103, 495 (2000).
}

${ }^{6}$ A. Koslowski, M. E. Beck, and W. Thiel, J. Comput. Chem. 24(6), 714 (2003).

${ }^{7}$ J. F. Stanton and R. J. Bartlett, J. Chem. Phys. 98, 7029 (1993).

${ }^{8}$ J. D. Watts and R. J. Bartlett, J. Chem. Phys. 101, 3073 (1994).

${ }^{9}$ H. Koch and P. Jørgensen, J. Chem. Phys. 93, 3333 (1990).

${ }^{10}$ H. Koch, H. J. A. Jensen, P. Jørgensen, and T. Helgaker, J. Chem. Phys. 93, 3345 (1990).

${ }^{11}$ R. J. Rico and M. Head-Gordon, Chem. Phys. Lett. 213, 224 (1993).

${ }^{12}$ J. Č́ížek, J. Chem. Phys. 45, 4256 (1966).

${ }^{13}$ J. Oddershede, Adv. Quantum Chem. 11, 275 (1978).

${ }^{14}$ J. Oddershede, Adv. Chem. Phys. 69, 201 (1987).

${ }^{15}$ B. O. Roos, P. R. Taylor, and P. E. M. Siegbahn, Chem. Phys. 48, 157 (1980).

${ }^{16}$ O. Christiansen, H. Koch, and P. Jørgensen, Chem. Phys. Lett. 243, 409 (1995).

${ }^{17}$ O. Christiansen, H. Koch, P. Jørgensen, and T. Helgaker, Chem. Phys. Lett. 263, 530 (1996).

${ }^{18}$ K. Hald, C. Hättig, D. L. Yeager, and P. Jørgensen, Chem. Phys. Lett. 328, 291 (2000).

${ }^{19}$ C. Hättig and F. Weigend, J. Chem. Phys. 113, 5154 (2000).

${ }^{20}$ C. Hättig, J. Chem. Phys. 118, 7751 (2003).

${ }^{21}$ A. Köhn and C. Hättig, J. Chem. Phys. 119, 5021 (2003).

${ }^{22}$ J. Oddershede and P. Jørgensen, J. Chem. Phys. 66, 1541 (1977).

${ }^{23}$ E. S. Nielsen and P. Jørgensen, J. Chem. Phys. 73, 6238 (1980).

${ }^{24}$ J. Oddershede, P. Jørgensen, and D. L. Yeager, Comput. Phys. Rep. 2, 33 (1984).

${ }^{25}$ J. Schirmer, Phys. Rev. A 26, 2395 (1982).

${ }^{26}$ A. B. Trofimov, G. Stelter, and J. Schirmer, J. Chem. Phys. 111, 9982 (1999).

${ }^{27}$ A. B. Trofimov, G. Stelter, and J. Schirmer, J. Chem. Phys. 117, 6402 (2002).

${ }^{28}$ S. Grimme and M. Parac, ChemPhysChem 4, 292 (2003).

${ }^{29}$ A. Dreuw and M. Head-Gordon, Chem. Rev. 105, 4009 (2005).

${ }^{30}$ T. Helgaker, P. Jørgensen, and J. Olsen, Molecular Electronic Structure Theory (Wiley, Chichester, 2000).

${ }^{31}$ R. J. Buenker and S. D. Peyerimhoff, Theor. Chim. Acta 35, 33 (1974).

${ }^{32}$ J. Schirmer, Phys. Rev. A 43, 4647 (1991).

${ }^{33}$ F. Mertins and J. Schirmer, Phys. Rev. A 53, 2140 (1996).

${ }^{34}$ J. Schirmer and A. B. Trofimov, J. Chem. Phys. 120, 11449 (2004).

${ }^{35}$ J. H. Starcke, M. Wormit, and A. Dreuw, J. Chem. Phys. 130, 024104 (2009).

${ }^{36}$ P. H. P. Harbach, M. Wormit, and A. Dreuw, J. Chem. Phys. 141, 064113 (2014).

${ }^{37}$ M. Wormit, D. R. Rehn, P. H. P. Harbach, J. Wenzel, C. M. Krauter, E. Epifanovsky, and A. Dreuw, Mol. Phys. 112, 774 (2014).

${ }^{38} \mathrm{M}$. Wormit and A. Dreuw, Wiley Interdiscip. Rev.: Comput. Mol. Sci. 5, 82 (2015).

${ }^{39}$ C. M. Krauter, M. Pernpointner, and A. Dreuw, J. Chem. Phys. 138, 044107 (2013).

${ }^{40}$ S. Knippenberg, D. R. Rehn, M. Wormit, J. H. Starcke, I. L. Rusakova, A. B. Trofimov, and A. Dreuw, J. Chem. Phys. 136, 064107 (2012).

${ }^{41}$ T. Fransson, D. R. Rehn, A. Dreuw, and P. Norman, J. Chem. Phys. 146, 094301 (2017).

${ }^{42}$ J. Wenzel, A. Holzer, M. Wormit, and A. Dreuw, J. Chem. Phys. 142, 214104 (2015).

${ }^{43}$ S. Mai, F. Plasser, M. Pabst, F. Neese, A. Köhn, and L. González, J. Chem. Phys. 147, 184109 (2017).

${ }^{44}$ D. Lefrancois, M. Wormit, and A. Dreuw, J. Chem. Phys. 143, 124107 (2015).

${ }^{45}$ S. Prager, A. Zech, F. Aquilante, A. Dreuw, and T. A. Wesolowski, J. Chem. Phys. 144, 204103 (2016).

${ }^{46}$ S. F. Boys, G. B. Cook, C. M. Reeves, and I. Shavitt, Nature 178, 1207 (1956).

${ }^{47}$ J. L. Whitten, J. Chem. Phys. 58, 4496 (1973).

${ }^{48}$ B. I. Dunlap, J. W. D. Connolly, and J. R. Sabin, J. Chem. Phys. 71, 3396 (1979).

${ }^{49}$ M. Feyereisen, G. Fitzgerald, and A. Komornicki, Chem. Phys. Lett. 208, 359 (1993).

${ }^{50}$ N. O. C. Winter and C. Hättig, J. Chem. Phys. 134, 184101 (2011).

${ }^{51}$ E. G. Hohenstein, R. M. Parrish, and T. J. Martínez, J. Chem. Phys. 137, 044103 (2012).

${ }^{52}$ R. M. Parrish, E. G. Hohenstein, T. J. Martínez, and C. D. Sherrill, J. Chem. Phys. 137, 224106 (2012). 
${ }^{53}$ E. G. Hohenstein, R. M. Parrish, C. D. Sherrill, and T. J. Martínez, J. Chem. Phys. 137, 221101 (2012).

${ }^{54}$ E. G. Hohenstein, S. I. L. Kokkila, R. M. Parrish, and T. J. Martínez, J. Chem. Phys. 138, 124111 (2013).

${ }^{55}$ E. G. Hohenstein, S. I. L. Kokkila, R. M. Parrish, and T. J. Martínez, J. Phys. Chem. B 117, 12972 (2013).

${ }^{56}$ R. Send, V. R. I. Kaila, and D. Sundholm, J. Chem. Phys. 134, 214114 (2011).

${ }^{57}$ R. Send, C.-M. Suomivuori, V. R. I. Kaila, and D. Sundholm, J. Phys. Chem. B 119, 2933 (2015).

${ }^{58}$ A. P. Gamiz-Hernandez, I. N. Angelova, R. Send, D. Sundholm, and V. R. I. Kaila, Angew. Chem., Int. Ed. 54, 11564 (2015).

${ }^{59}$ C.-M. Suomivuori, N. O. C. Winter, C. Hättig, D. Sundholm, and V. R. I. Kaila, J. Chem. Theory Comput. 12, 2644 (2016).

${ }^{60}$ C. Yang and A. Dreuw, J. Comput. Chem. 38, 1528 (2017).

${ }^{61}$ P. Pulay, Chem. Phys. Lett. 100, 151 (1983).

${ }^{62}$ P. Pulay and S. Saebø, Theor. Chim. Acta 69, 357 (1986).

${ }^{63}$ M. Schütz, J. Chem. Phys. 142, 214103 (2015).

${ }^{64}$ B. Helmich and C. Hättig, Comput. Theor. Chem. 1040-1041, 35 (2014).

${ }^{65}$ D. Kats, T. Korona, and M. Schütz, J. Chem. Phys. 125, 104106 (2006).

${ }^{66}$ D. Kats, T. Korona, and M. Schütz, J. Chem. Phys. 127, 064107 (2007).

${ }^{67}$ D. Kats and M. Schütz, J. Chem. Phys. 131, 124117 (2009).

${ }^{68} \mathrm{~K}$. Freundorfer, D. Kats, T. Korona, and M. Schütz, J. Chem. Phys. 133, 244110 (2010).

${ }^{69}$ K. Ledermüller, D. Kats, and M. Schütz, J. Chem. Phys. 139, 084111 (2013).

${ }^{70}$ K. Ledermüller and M. Schütz, J. Chem. Phys. 140, 164113 (2014).

${ }^{71}$ P. Baudin and K. Kristensen, J. Chem. Phys. 144, 224106 (2016).

${ }^{72}$ P. Baudin and K. Kristensen, J. Chem. Phys. 146, 214114 (2017).

${ }^{73}$ W. Meyer, J. Chem. Phys. 58, 1017 (1973).

${ }^{74}$ P. J. Hay, J. Chem. Phys. 59, 2468 (1973).

${ }^{75}$ R. Ahlrichs, H. Lischka, V. Staemmler, and W. Kutzelnigg, J. Chem. Phys. 62, 1225 (1975)

${ }^{76}$ A. G. Taube and R. J. Bartlett, J. Chem. Phys. 128, 164101 (2008).

${ }^{77}$ A. E. DePrince and C. D. Sherrill, J. Chem. Theory Comput. 9, 293 (2013).

${ }^{78}$ Z. Rolik and M. Kállay, J. Chem. Phys. 134, 124111 (2011).

${ }^{79}$ Z. Rolik, L. Szegedy, I. Ladjánszki, B. Ladóczki, and M. Kállay, J. Chem. Phys. 139, 094105 (2013).

${ }^{80}$ A. Landau, K. Khistyaev, S. Dolgikh, and A. I. Krylov, J. Chem. Phys. 132, 014109 (2010).

${ }^{81}$ A. Kumar and T. D. Crawford, J. Phys. Chem. A 121, 708 (2017).

${ }^{82}$ R. Flores-Moreno and J. V. Ortiz, J. Chem. Phys. 128, 164105 (2008).

${ }^{83}$ O. Dolgounitcheva, R. Flores-Moreno, V. G. Zakrzewski, and J. V. Ortiz, Int. J. Quantum Chem. 108, 2862 (2008).

${ }^{84}$ V. G. Zakrzewski, O. Dolgounitcheva, A. V. Zakjevskii, and J. V. Ortiz, Annu. Rep. Comput. Chem. 6, 79 (2010).

${ }^{85}$ M. Kállay, J. Chem. Phys. 141, 244113 (2014).

${ }^{86}$ D. Mester, P. R. Nagy, and M. Kállay, J. Chem. Phys. 146, 194102 (2017).

${ }^{87}$ P. Pulay, Chem. Phys. Lett. 73, 393 (1980).

${ }^{88}$ B. Lunkenheimer and A. Köhn, J. Chem. Theory Comput. 9, 977 (2013).
${ }^{89}$ M. Pabst and A. Köhn, J. Chem. Phys. 129, 214101 (2008).

${ }^{90}$ M. Head-Gordon, R. J. Rico, M. Oumi, and T. J. Lee, Chem. Phys. Lett. 219, 21 (1994).

${ }^{91}$ G. E. Scuseria, A. C. Scheiner, T. J. Lee, J. E. Rice, and H. F. Schaefer III, J. Chem. Phys. 86, 2881 (1987).

${ }^{92}$ M. Kállay, Z. Rolik, J. Csontos, P. Nagy, G. Samu, D. Mester, J. Csóka, I. Ladjánszki, L. Szegedy, B. Ladóczki, K. Petrov, M. Farkas, and B. Hégely, MRCC, a quantum chemical program suite, see also Ref. 79 as well as http://www.mrcc.hu/.

${ }^{93}$ T. H. Dunning, Jr., J. Chem. Phys. 90, 1007 (1989).

${ }^{94}$ R. A. Kendall, T. H. Dunning, Jr., and R. J. Harrison, J. Chem. Phys. 96, 6796 (1992).

${ }^{95}$ D. E. Woon and T. H. Dunning, Jr., J. Chem. Phys. 98, 1358 (1993).

${ }^{96} \mathrm{~F}$. Weigend, A. Köhn, and C. Hättig, J. Chem. Phys. 116, 3175 (2002).

${ }^{97}$ F. Weigend, M. Häser, H. Patzelt, and R. Ahlrichs, Chem. Phys. Lett. 294, 143 (1998).

${ }^{98}$ F. Weigend, J. Comput. Chem. 29, 167 (2008).

${ }^{99}$ M. Schreiber, M. R. Silva-Junior, S. P. A. Sauer, and W. Thiel, J. Chem. Phys. 128, 134110 (2008).

${ }^{100}$ M. R. Silva-Junior, S. P. A. Sauer, M. Schreiber, and W. Thiel, Mol. Phys. 108, 453 (2010).

${ }^{101}$ A. Dreuw, J. L. Weisman, and M. Head-Gordon, J. Chem. Phys. 119, 2943 (2003).

${ }^{102}$ M. Parac and S. Grimme, J. Phys. Chem. A 106, 6844 (2002).

${ }^{103}$ L. Goerigk, J. Moellmanna, and S. Grimme, Phys. Chem. Chem. Phys. 11, 4611 (2009).

${ }^{104}$ L. Goerigk and S. Grimme, J. Chem. Phys. 132, 184103 (2010).

${ }^{105}$ A. Matsuura, H. Sato, W. Sotoyama, A. Takahashi, and M. Sakurai, J. Mol. Struct.: THEOCHEM 860, 119 (2008).

${ }^{106}$ D. Jacquemin, V. Wathelet, E. A. Perpéte, and C. Adamo, J. Chem. Theory Comput. 5, 2420 (2009).

${ }^{107}$ D. Jacquemin, E. A. Perpéte, I. Ciofini, C. Adamo, R. Valero, Y. Zhao, and D. G. Truhlar, J. Chem. Theory Comput. 6, 2071 (2010).

${ }^{108}$ D. Jacquemin, I. Duchemin, and X. Blase, J. Chem. Theory Comput. 11, 5340 (2015).

${ }^{109}$ D. A. Yushchenko, O. B. Vadzyuk, S. O. Kosterin, G. Duportail, Y. Mély, and V. G. Pivovarenko, Anal. Biochem. 369, 218 (2007).

${ }^{110}$ C. A. Kenfack, A. S. Klymchenko, G. Duportail, A. Burgerc, and Y. Mély, Phys. Chem. Chem. Phys. 14, 8910 (2012).

${ }^{111} \mathrm{O}$. Christiansen, H. Koch, P. Jørgensen, and J. Olsen, Chem. Phys. Lett. 256, 185 (1996).

${ }^{112}$ H. Koch, O. Christiansen, P. Jørgensen, and J. Olsen, Chem. Phys. Lett. 244, 75 (1995).

${ }^{113}$ C. Hättig, Adv. Quantum Chem. 50, 37 (2015).

${ }^{114}$ N. O. C. Winter, N. K. Graf, S. Leutwyler, and C. Hättig, Phys. Chem. Chem. Phys. 15, 6623 (2013).

${ }^{115}$ R. Send, M. Kühn, and F. Furche, J. Chem. Theory Comput. 7, 2376 (2011).

${ }^{116}$ S. Budzák, G. Scalmani, and D. Jacquemin, J. Chem. Theory Comput. 13, 6237 (2017). 